\title{
Resonant Tunnelling Optoelectronic Circuits
}

\author{
José Figueiredo ${ }^{1}$, Bruno Romeira ${ }^{1}$, Thomas Slight ${ }^{2}$ and Charles Ironside ${ }^{2}$ \\ ${ }^{1}$ Centro de Electrónica, Optoelectrónica e Telecomunicacões, Universidade do Algarve \\ ${ }^{2}$ Department of Electronics and Electrical Engineering, University of Glasgow \\ ${ }^{1}$ Portugal \\ ${ }^{2}$ United Kingdom
}

\section{Introduction}

Nowadays, most communication networks such as local area networks (LANs), metropolitan area networks (MANs), and wide area networks (WANs) have replaced or are about to replace coaxial cable or twisted copper wire with fiber optical cables. Light-wave communication systems comprise a transmitter based on a visible or near-infrared light source, whose carrier is modulated by the information signal to be transmitted, a transmission media such as an optical fiber, eventually utilizing in-line optical amplification, and a receiver based on a photo-detector that recovers the information signal (Liu, 1996)(Einarsson, 1996). The transmitter consists of a driver circuit along a semiconductor laser or a light emitting diode (LED). The receiver is a signal processing circuit coupled to a photo-detector such as a photodiode, an avalanche photodiode (APD), a phototransistor or a high speed photoconductor that processes the photo-detected signal and recovers the primitive information signal.

Transmitters and receivers are classical examples of optoelectronic integrated circuits (OEICs) (Wada, 1994). OEIC technologies aim to emulate CMOS microelectronics by (i) integrating optoelectronic devices and electronic circuitry on the same package or substrate (hybrid integration), (ii) monolithically integrate III-V optoelectronic devices on silicon (difficulty since silicon is not useful for many optoelectronic functions) or (iii) monolithically integrate III-V electronics with optoelectronic devices. The simply way to do hybrid integration is combining packaged devices on a ceramic substrate. More advanced techniques include flip-chip/solder-ball or -bump integration of discrete optoelectronic devices on multi-chip modules or directly on silicon integrated circuit (IC) chips, and flipbonding on IC chips. Although, hybrid integration offers immediate solutions when many different kinds of devices need to be combined it produces OEICs with very low device density. Moreover, in certain cases the advantages of using optical devices is greatly reduced. On the contrary, monolithic integration leads to superior speed, component density, reliability, complexity, and manufacturability (Katz, 1992).

There was been substantial efforts towards monolithical integration of III-V electronics with optoelectronic devices to improve the performance of transmitters and receivers. Approaches to light modulation, light detection and light generation at microwave and millimetre-wave frequencies have been investigated by combining double barrier quantum well (DBQW) resonant tunnelling diodes (RTDs) with optical components such as 
waveguides (Figueiredo, 2000) and semiconductor lasers (Slight, 2006). These RTD based OEICs can operate as novel optoelectronic voltage controlled oscillators (OVCOs), with potential to simplify clock recovery circuits, improve control of microwave oscillators functionalities, to generate electrical and optical aperiodic waveforms, and as microwave-tooptical subcarrier and optical subcarrier-to-microwave converters for radio-over-fiber systems, where the integration of electrical and optical components in a single chip is a major challenge in order to obtain high reliability, small size and low cost (Sauer et al., 2007).

This chapter reports investigation on resonant tunnelling (RT) based OEICs that demonstrate new functionalities for optical modulators and sources for application in telecommunication systems and signal processing circuits. Section 2 starts with a brief description of DBQW-RTD's operating principle, followed by the presentation of a physics based model of its current-voltage $(I-V)$ characteristic, continues with a small-signal equivalent circuit analysis, and ends with an overview of more relevant optoelectronic devices incorporating RT structures. Section 3 describes the integration of DBQW-RTDs within an optical waveguide (OW) towards the implementation of very low driving voltage electro-absorption modulators (EAMs) and optical detectors (OD), with built-in amplifiers, for operation at optical wavelengths around $900 \mathrm{~nm}$ and $1550 \mathrm{~nm}$. Section 4 discusses monolithic and hybrid integration of a DBQW-RTD with a laser diode (LD), its operation principle and optoelectronics circuit model used to analyse its modes of operation including optoelectronic voltage controlled oscillator (OVCO), frequency division and multiplication, phase-locking, and the generation of aperiodic, even chaotic, waveforms. The chapter ends with conclusion and acknowledgement sections.

\section{Resonant tunnelling diode}

Resonant tunnelling diodes (RTDs) are nanoelectronic structures that can be easily integrated with conventional electronic and photonic devices (Davies, 1998)(Mizuta \& Tanoue, 1995)(Sun et al., 1998), such as transistors (Mazumder et al., 1998), optical waveguides (McMeekin et al., 1994)(Figueiredo, 2000) and laser diodes (Slight, 2006) with potential to not only reduce power consumption and cost but also increase functionality, speed and circuit reliability, without losing any advantage of using optical devices. They have two distinct features when compared with other semiconductor devices (Mazumder et al., 1998): their potential for extremely high frequency operation up to terahertz and their negative differential conductance (NDC). The former arises from the very small size of the resonant tunnelling structure along the direction of carriers transport. The second corresponds to electric gain which makes possible to operate RTDs as amplifiers and oscillators, significantly reducing the number of elements required for a given function (Mazumder et al., 1998). Functional RTD based devices and circuits span from signal generators, detectors and mixers, multi-valued logic switches, low-power amplifiers, local oscillators, frequency locking circuits, and also as generators of multiple high frequency harmonics (Mizuta \& Tanoue, 1995). In this section, the physics of double barrier quantum well resonant tunnelling diodes (DBQW-RTDs) is discussed and analyzed, aiming at its application in high speed optoelectronic converters (rf-optical and optical-rf), such as light emitters, light modulators and light detectors. 


\subsection{Double barrier quantum well RTD}

Resonant tunnelling through double potential barriers was predicted by (Bohm, 1951). Latter, (Iogansen, 1964) discussed the possibility of resonant transmission of an electron through double barriers formed in semiconductor crystals. They concluded that structures with identical barriers show tunnelling transmission coefficients of 1 when the particles incident energy equals the structure resonant energies, however small the transmission through the individual barriers may be (Mizuta \& Tanoue, 1995). Figure 1 compares schematically the transmission coefficient $T(E)$ for single and symmetrical double barrier structures. The transmission coefficient lobs broadens with increasing energy because the barriers become more transparent (Davies, 1998).

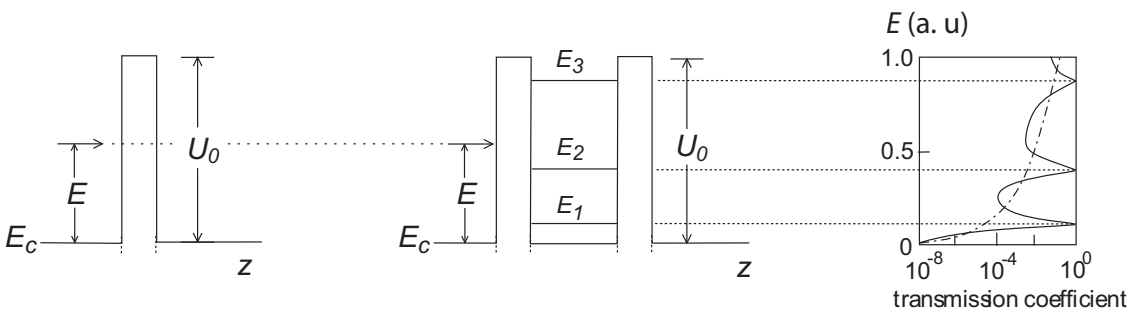

Fig. 1. Single and DBQW transmission coefficients as function of incident carrier energy.

A semiconductor double barrier quantum well resonant tunnelling diode (DBQW-RTD) consists of a low band-gap semiconductor layer (the quantum well, typical $5 \mathrm{~nm}$ to $10 \mathrm{~nm}$ wide) surrounded by two thinner layers of higher band-gap material (barriers, typical 1.5 $\mathrm{nm}$ to $5 \mathrm{~nm}$ ), both sandwiched between low band-gap $n$-type material layers, typical the well material, as schematically shown in Fig. 2(a) (Mizuta \& Tanoue, 1995). The material forming the barriers must have a positive conduction-band offset with respect to the smaller bandgap materials (Weisbuch \& Vinter, 1991). When both sides are terminated by highly doped semiconductor layer (the emitter and the collector contacts) for electrical connection the structure is called resonant tunnelling diode (RTD). Figure 2(b) shows a schematic of a ntype Al-GaAs/GaAs DBQW-RTD, together with the $\Gamma$-conduction band profiles at around zero volts and at the peak voltage. Because finite height of the energy barriers the allowed energy states in the well region become quasi-bound or resonant states, Fig. 2(a), rather than true bound states as it happens with thicker barrier quantum wells (Davies, 1998). In consequence, tunneling of charge carriers through the barriers is strongly enhanced when their energy equals to one of well energy levels, reaching much higher values than the product of the two individual barrier transmission coefficients at the energy values of the system resonant levels, see Fig. 1.

(a)

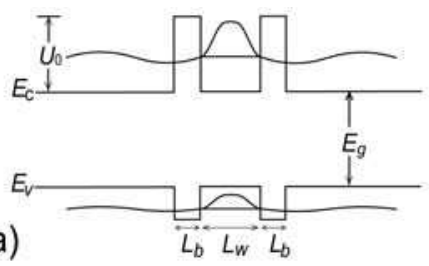

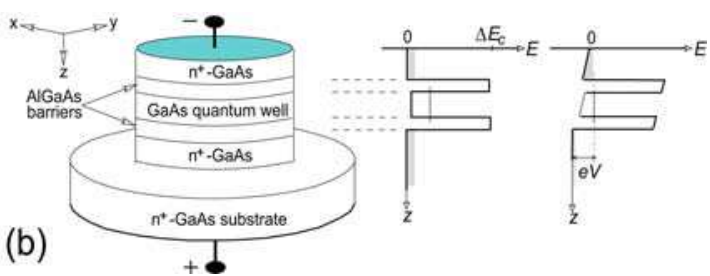

(b)

Fig. 2. (a) DBQW semiconductor structure. (b) AlGaAs DBQW structure (left); $\Gamma$-conduction band profiles at zero and at the first resonance voltage (right). 
Under applied bias, the overall carrier flow through a DBQW-RTD is qualitatively different from that of a single barrier diode since the double barrier structure acts as a band filter to charge carrier energy distribution (Mizuta \& Tanoue, 1995)(Sun et al., 1998). This filter action is exploited applying a voltage across the DBQW structure to control the number of carriers that can take part in the conduction through resonant levels. The carrier transmission coefficient maxima shown in Fig. 1 give rise to current-voltage characteristics with regions of strong NDC. The resonant tunnelling phenomenon in AlGaAs DBQW structures was first predicted in 1973 (Tsu \& Esaki, 1973), and demonstrated experimentally in 1974 (Chang et al., 1974). In 1983, Sollner et al. demonstrated resonant tunnelling through quantum wells at frequencies up to $2.5 \mathrm{THz}$ (Sollner et al., 1983). Figure 3(a) shows a typical InGaAs/AlAs RTD I - V characteristic. The main carrier flow processes in a DBQW-RTD polarized at the peak voltage (the current first maxima) is schematically represented in Fig. 3(b).

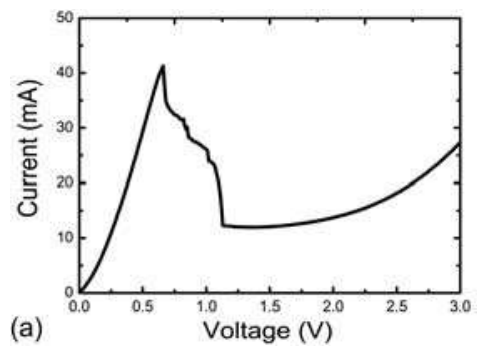

Fig. 3. (a) Typical InGaAlAs RTD I-V characteristic. (b) Current transport mechanisms in DBQW-RTDs at the peak voltage (Sun et al., 1998).

The RTD current-voltage characteristic of Fig. 3(a) can be understood with the help of the $\Gamma$ conduction band profile shown in Figs. 2(b) and 3(b) (Davies, 1998). When the applied bias is small, i.e., $V \ll V_{p}$ (peak voltage, also referred as resonance voltage), the $\Gamma$-conduction band profile is not much affected, remaining almost flat, see Fig. 2(b). The first resonant level is well above the emitter Fermi level, and little current flows. As voltage is increased, the energy of the first resonant level is moved downwards to the emitter Fermi level, leading to an almost linearly current increase with the voltage, the first positive differential conductance (PDC) region, till reaching a local maximum $I_{p}$, ideally, at $V \simeq 2 E_{n=1} / e$, when the overlap between the emitter electron Fermi sea energy spectrum and the transmission coefficient around the first resonant level reaches a local maximum, as shown in the right side of Fig. 2(b) and Fig. 3(b). A further increase in the applied voltage pulls the first resonant level towards the bottom of the $\Gamma$-valley and into the forbidden gap, where there are no longer carriers available to efficiently cross the DBQW. This leads to a sharp current decrease, giving rise to the first negative differential conductance (NDC) portion of the device current-voltage characteristic. At a given voltage, known as the valley voltage $V_{v}$, with $V_{v}>V_{p}$, the current reaches a local minimum $I_{v}$. An additional increase on the bias voltage will further lift up the emitter Fermi level and tunnelling through higher resonant levels or through the top regions of the barriers will lead to new current rise, similar to the classical diode $I-V$ characteristic (Davies, 1998). The resonant tunnelling component dominates at low voltages and the classical diode component takes over at higher voltages. For more details see (Davies, 1998)(Sun et al., 1998). In a circuit, the NDC provides the gain necessary to sustain oscillations (Mizuta \& Tanoue, 1995) (Brown \& Parker 1996). The 
presence of a small inductance in circuit containing an RTD, together with RTD intrinsic capacitance make possible the oscillations at very high frequencies, experimental demonstrated up to $831 \mathrm{GHz}$ (Suzuki et al., 2009). Frequencies never reached by other semiconductor devices: the RTD is currently the fastest purely electronic device.

The most common material systems used to implement RTD devices are III-V compounds such as AlGaAs and InP-based materials.. Si/SiGe RTDs based on Si/SiGe heterojunctions have been demonstrated but the performance is not comparable to III-V RTDs because of the limited band edge discontinuity in both valence and conduction bands. Organic RTDs are currently being investigated (Park et al., 2006)(Ryu et al., 2007)(Zheng et al., 2009).

\subsection{RTD based generalized Liénard oscillator}

The RTDs inherent high speed operation, up to terahertz frequency, the pronounced nonlinear current-voltage characteristic, wide-bandwidth NDC, structural simplicity, flexible design, relative ease of fabrication, and versatile circuit functionality, make them excellent candidates for nanoelectronic circuit applications. In order to take advantage of the full potential of RTD based devices several attempts have been made to incorporate the full RTD characteristics into circuit simulation packages such as SPICE-like CAD tools (Mizuta \& Tanoue, 1995)(Brown et al., 1996)(Sun et al., 1998).

Since a quantum mechanics based model that includes all RTD features is not yet available, a number of empirical models have been advanced (Sun et al., 1998). Most models describe the RTD by small-signal equivalent circuits consisting of a capacitance $C$, resulting from charging and discharging of electrons of DBQW and depletion regions, in parallel with a voltage depend current source $I=F(V)$, a series resistance $R$ arising mainly from the ohmic contacts and an inductance $L$ due to bond wire connections, Fig. 4 . The current source $F(V)$ is usually implemented as polynomial or piecewise functions (Brown et al., 1997)(Sun et al., 1998), which is not satisfactory if a detailed circuit description is needed. More useful RTD non-linear characteristic representations have to consider a wide variety of device structures and the materials available, i.e., the modelled $I-V$ characteristic has to be based as much as possible on the RTD physical parameters such as material properties, layer dimensions, energy levels, dopant concentrations, and the device geometry.

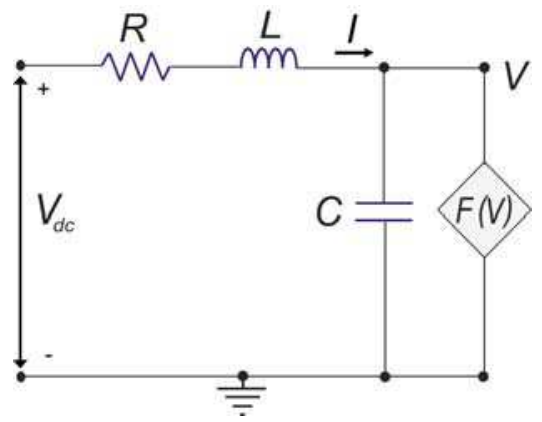

Fig. 4. Electrical equivalent circuit of an RTD represented by a capacitance in parallel with a voltage dependent current source $F(V)$. The inductance $L$ and the resistor $R$ are due to bonding wires and contacts.

The physics based model proposed by Schulman et al. consists of a mathematical function which provides a satisfactory $I-V$ shape characteristic for InGaAs and GaAs RTD based 
devices (Schulman et al., 1996). The expression obtained contains physical quantities which can also be treated as empirical parameters for fitting purposes. In their analysis the resonant tunnelling current density is expressed within the effective mass approximation (Davies, 1998), which includes nonzero temperature, Fermi-Dirac statistics and the transmission coefficient $T(E, V)$ :

$$
J_{R T}=\frac{q m^{*} k_{B} T \cdot \Delta E_{r}}{4 \pi^{2} \hbar^{3}} \ln \left[\frac{1+e^{\left(E_{F}-E_{r}+q V / 2\right) / k_{B} T}}{1+e^{\left(E_{F}-E_{r}-q V / 2\right) / k_{B} T}}\right] \cdot\left[\frac{\pi}{2}+\tan ^{-1}\left(\frac{E_{r}-q V / 2}{\Delta E_{r} / 2}\right)\right]
$$

where $E=E_{r}-q V / 2$ is the energy measured up from the emitter conduction band edge, $E_{r}$ is the energy of the resonant level relative to the bottom of the well at its centre, and $\Delta E_{r}$ is the resonance width. The parameters $q$ and $k_{B}$ are unit electric charge and Boltzmann constants, respectively. Equation 1 can be rewritten as:

$$
J_{R T}(V)=A \cdot \ln \left[\frac{1+e^{q\left(B-C+n_{1} V\right) / k_{B} T}}{1+e^{q\left(B-C-n_{1} V\right) / k_{B} T}}\right] \cdot\left[\frac{\pi}{2}+\tan ^{-1}\left(\frac{C-n_{1} V}{D}\right)\right]
$$

where the parameters $A, B, C, D$, and $n_{1}$ can be used to shape the curve to match the first PDC region of the measured $I-V$ characteristic, having at the same time a well-defined physical interpretation: $A$ and $B$ are related, among other factors, with resonance width and Fermi level energies, and allow adjustment of the RTD peak current; $C$ and $n_{1}$ determine essentially the RTD peak voltage, correlated with the energy of the resonant level relative to the bottom of the well and with the transmission coefficient; finally, $D$ is related to the resonance width $\Delta E_{r}$.

In order to represent the increasing valley current due to tunnelling through higher resonances or thermal excitation over the barriers, an additional current density component, identical to the classical diode current, the non-resonant term $J_{N R}$, have to be included:

$$
J_{N R}(V)=H\left(e^{n_{2} q V / k_{B} T}-1\right)
$$

Parameters $D$ and $H$ adjustment of adjust the peak to valley current ratio (PVCR) and the peak to valley voltage ratio (PVVR).

Equations 2 and 3 give good estimations of the peak current and the NDC region of currentvoltage characteristic. The final form of the RTD current-voltage curve is then given by:

$$
I(V)=I_{R T}(V)+I_{N R}(V)=M\left[J_{R T}(V)+J_{N R}(V)\right]
$$

where the multiplying factor $M$ is used to scale equation 4 , in order to take into account the devices area. Figure 5 shows experimental $I-V$ curves of AlGaAs (a), and InGaAlAs (b), RTDs, with the corresponding fit given by equation 4 . The fits assumed operation at temperature $T=300 \mathrm{~K}$ and a multiplying factor $M=2 \times 10^{-6} \mathrm{~cm}^{2}$, with the following parameters: $A=1950 \mathrm{~A} / \mathrm{cm}^{2}, B=0.05 \mathrm{~V}, \mathrm{C}=0.0874 \mathrm{~V}, D=0.0073 \mathrm{~V}, n_{1}=0.0352, H=18343 \mathrm{~A} / \mathrm{cm}^{2}$, and $n_{2}=0.0031$ for AlGaAs; $A=3800 \mathrm{~A} / \mathrm{cm}^{2}, B=0.068 \mathrm{~V}, C=0.1035 \mathrm{~V}, D=0.0088 \mathrm{~V}, n_{1}=0.0862$, $H=4515 \mathrm{~A} / \mathrm{cm}^{2}$, and $n_{2}=0.0127$ for InGaAlAs. Higher values of $A$ and $B$ are used in the InGaAlAs fitting due to RTD higher peak current; parameter $D$ was also slightly larger for the InGaAlAs due to superior PVCR and PVVR. The parameter $H$ was around four times larger in the AlGaAs due mainly to their higher peak voltages. 

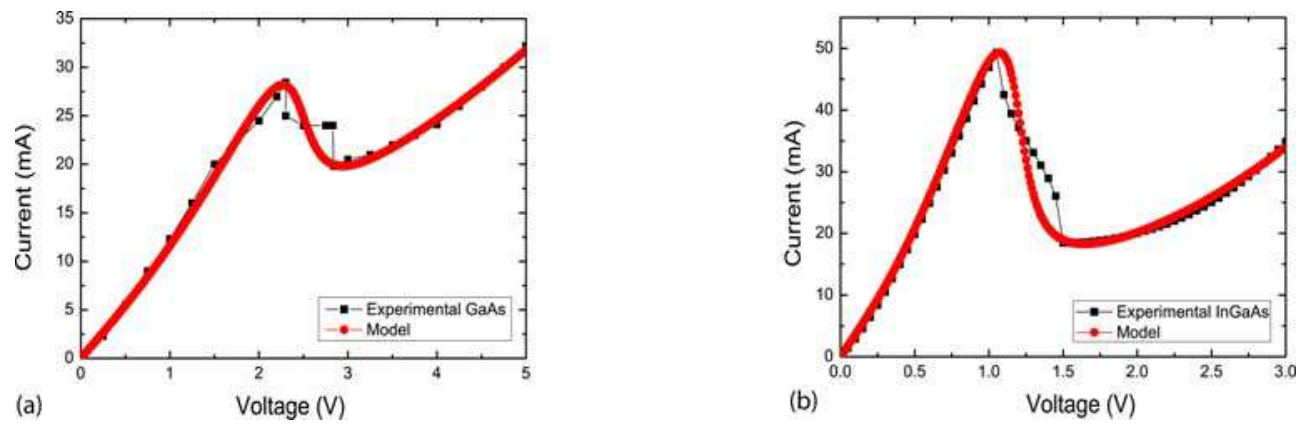

Fig. 5. GaAs/AlAs (a) and InGaAs/AlAs (b) RTD experimental $I$ - Vs and fittings.

Since the RTD is a voltage-dependent current source device, when incorporated in a resonant circuit and biased in the NDC portion of its $I-V$ characteristic produces oscillations at circuit characteristic frequency (Brown \& Parker, 1996). In order to understand the origin of the circuit self-oscillations induced by the RTD we consider the small-signal equivalent circuit of Fig. 4. Typical RTD switching times are in general dominated by the effects of current densities and capacitances, i.e., by the circuit $R C$ time constant (Brown et al., 1997) (Brown \& Parker, 1996).

A general analysis of a circuit containing an RTD considers the small signal equivalent circuit of Fig. 4, where the RTD non-linear $I-V$ characteristic is represented by a voltage dependent current source $F(V)$, given by equation 4 , in parallel with RTD intrinsic capacitance $C$. Resistor $R$ and inductor $L$ encompasses for the device series resistance and connections inductance, respectively. By applying Kirchoff's laws (using Faraday's law) to the circuit of Fig. 4, the voltage $V$ across the capacitance $C$ and the current $I$ through the inductor $L$ are given by the following set of two first-order non-autonomous differential equations (Slight et al., 2008):

$$
\begin{gathered}
\dot{V}=\frac{1}{C}[I-F(V)] \\
\dot{I}=\frac{1}{L}\left(V_{d c}-R I-V\right)
\end{gathered}
$$

After some algebra, we find that the system of Eqs. 5-6 is equivalent to the following secondorder differential equation, referred as one of the generalized nonlinear Liénard systems (Slight et al., 2008)(Figueiredo, 1970):

$$
\begin{gathered}
\ddot{V}+\left[\frac{R}{L}+\frac{1}{C} \frac{d F(V)}{d V}\right] \dot{V}+\frac{1}{L C}\left[V-V_{d c}+R F(V)\right]=0 \\
\ddot{V}+H(V) \dot{V}+G(V)=0
\end{gathered}
$$

"where $H(V)=\frac{R}{L}+\frac{1}{C} \frac{d F(V)}{d V}$ and $G(V)=\frac{1}{L C}\left[V-V_{d c}+R F(V)\right] . G(V)$ is a nonlinear force and $H(V) \dot{V}$ is a damping factor. 
The circuit of Fig. $4 \mathrm{dc}$ biased in the NDC acts as a relaxation oscillator producing oscillations at a frequency around $f_{0}(V) \approx(2 \pi \sqrt{L \cdot C(V)})^{-1}$, the circuit characteristic frequency, whenever the series $R$ is smaller than the RTD operating point negative differential resistance (Brown \& Parker, 1996). From the application point of view the wideband NDC of RTD leads to low frequency oscillations instabilities that are detrimental. A most common source of instability arises from the dc source circuitry by introducing in the circuit an equivalent inductance, which together with RTD capacitance leads to oscillations at around few megahertz (Figueiredo, 2000)(Slight, 2006). A method to eliminate these low frequency oscillations and allowing circuit operation at much higher frequency is to place a shunt capacitor across the terminals of the device (Kidner et al., 1990)(Huang et al., 1997). The inductance is now only due to the connection from the shunt capacitor to the RTD.

\subsection{Optoelectronic applications of RT structures}

Several optoelectronic devices and circuits whose functions depend on embedded resonant tunnelling structures have been proposed and demonstrated, including resonant tunneling light emitting diodes (RT-LEDs) (Van Hoof et al., 1992), vertically integrated semiconductor lasers with RTDs (Grave et al., 1991), resonant tunnelling effect quantum-well lasers (Kawamura et al., 1994), resonant tunnelling injection laser (Capasso et al., 1986), multiquantum well (MQW) lasers (Kawamura et al., 1987) and photo-detecting (PD) structures (Chen et al., 1991). The nature and the energies involved in the carrier transition induced by the light interaction with the tunnelling layers determine the operation in the optical or in the infrared part of the electromagnetic spectrum. Optical applications such as photodetection, light emission, optical switching, utilize inter-band transitions (band-gap transitions), whereas infrared applications include intra-band and inter-sub-band photodetection, and infrared emission. Below is presented a brief summary of the main progress on optical and optoelectronic devices whose functionalities depend of embedded RT structures.

Bistability in the light output of bipolar RT-LEDs has been reported, showing that these devices are capable of ultrafast optical switching and high frequency optical oscillation (Van Hoof et al., 1993). Laser transistors incorporating a resonant tunnelling structure have been reported, with carrier injection or extraction controlled via resonant tunnelling structure, with light output controlled by the collector voltage and achieving higher speed than with conventional semiconductor lasers (Kawamura et al., 1992). Embedding RTs into multiquantum well (MQW) devices introduces negative differential conductance over wide valley region, which is very effective for getting large voltage switching and high on/off ratio current switching (Kawamura et al., 1988) leading to electro-optic bistability (Chen et al., 1991). Optical bistability in QW lasers integrated with DBQW-RTDs, and a RTD with a MQW modulator/detector based on the $p-i(\mathrm{MQW})-n$ configuration, operating at room temperature, were reported (Kawamura et al., 1994). Clear negative differential conductance and bistability, with high contrast and high sensitivity in resonant tunnelling triangular barrier optoelectronic switch (R-TOPS), which consists of a double barrier resonant tunnelling diode and a triangular barrier phototransistor has been demonstrated (Sakata et al., 1995).

A light pulse incident upon a resonant tunnelling diode produces photo-charges that reduce the series resistance, leading to a shift of the peak and valley voltages which can induce RTD 
switching and give rise to changes in the current flow (Moise et al., 1995). Optically switched resonant tunnelling diode (ORTD) photo-detectors have been demonstrated (Moise et al., 1997). Phase locking of an oscillating GaAs/AlGaAs RTD to a train of light pulses achieved by direct illumination was reported (Lann et al., 1993), as well as optical switching in resonant tunnelling diode (England et al., 1991) and optical injection locking of the resonant tunnelling oscillator (Kan et al., 2001). The RT structures can be used to implement light-by-light switching (England et al., 1991). Ultra-fast optoelectronic circuits using RTDs and uni-travelling-carrier photodiodes (UTC-PDs) to de-multiplex ultra-fast optical data signals into electrical data signals with lower bit rate and low power consumption has been demonstrated (Sano et al., 1998).

Our work on optoelectronic devices based on the integration of a RTD within an optical waveguide, and on hybrid and monolithic integrations of RTDs with laser diodes is discussed in the remaining sections of this chapter.

\section{RTD optical waveguide modulator-photodetector}

Novel information and communication technologies relying on microwave/millimetrewavelightwave interactions are fundamental to the development of applications such as low-cost fibre-optic communication networks, cable television signal distribution, mobile communications, and radio local area networks (Sauer et al., 2007). In this section, electrical active, high speed, highly efficient and low-cost electro-absorption modulators and photodetectors based on the integration of a RT structure within a semiconductor optical waveguide are described.

\subsection{RTD optical waveguide integration}

As discussed previously, when the RTD is biased in the valley region most of the applied voltage is dropped across the depletion region formed between the second barrier and the collector contact, Fig. 6(a), where a strong electric field builds-in. Inter-band electroabsorption of light with photon energies close to but smaller than the collector band-gap energy is achieved through the Franz-Keldysh effect (Chuang, 1995). According to the Franz-Keldysh effect the semiconductor material optical absorption band-edge is broadened by the presence of an electric field, resulting in an increase of absorption of light with photon energies smaller but close to the material band-gap (Keldysh, 1958). This effect is used to implement either electro-absorption (EAM) (intensity) modulators (Wakita et al., 1998) or waveguide photo-detectors (Chuang, 1995). However, in typical RTD structures the light is injected perpendicularly to the tunnelling plane, which gives a light interaction (absorption) length well below $100 \mathrm{~nm}$, and thus very small light absorption. This limitation can be easily overcome embedding the RTD into the core of a unipolar semiconductor optical waveguide (McMeekin et al., 1994). A typical waveguide structure is represented schematically in Fig. 6(a), showing also wafer $\Gamma$-conduction band-edge and refractive index profiles. This optoelectronic device is called resonant tunnelling diode optical waveguide (RTD-OW). The waveguide refractive index distribution confines light end-fire coupled along the tunnelling layers and the collector depleted region, therefore increasing substantially the light interaction volume along the waveguide length as indicated in Fig. 6(b). The RTD-OW, apart from the light confining layers (the lower refractive index regions upper and lower cladding layers), corresponds to a DBQW-RTD with thick low doped 
(a)
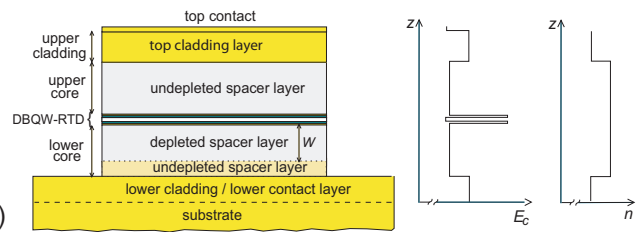

(b)

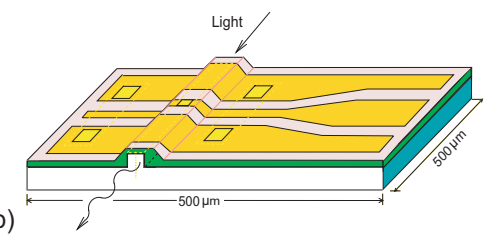

Fig. 6. (a) Diagram of a unipolar resonant tunnelling diode optical waveguide (RTD-OW) wafer structure, and the corresponding $\Gamma$-conduction band-edge and refractive index profiles. (b) Ridged waveguide channel configuration.

emitter and collector spacer layers. The presence of the DBQW within the waveguide core modifies the unipolar waveguide linear current-voltage characteristic towards the DBQWRTD strong nonlinear $I-V$ curve (McMeekin et al., 1994)(Figueiredo, 2000). Moreover, it leads to a non-linear electric field distribution across the collector side waveguide core that is strongly dependent on the bias voltage, due to the electron accumulation close to the emitter barrier and the creation of a depletion region on the collector spacer layer. Since a small voltage can be used to make a RTD operating point to switch between peak and valley regions, the RTD-OW can be employed to implement electro-absorption modulators (McMeekin et al., 1994)(Figueiredo, 2000). A small voltage change results in large modulation of the electric field across the device collector depletion region, resulting, though the Franz-Keldysh effect, in waveguide propagation losses and electro-absorption for photon energies close to but smaller than the waveguide core band-gap energy (Figueiredo, 2000)(Figueiredo et al., 2001).

The RTD-OW electric field distribution dependence on the bias voltage can be understood by considering the $\Gamma$-conduction band profile of the collector spacer layer, Fig. 7. Below resonance (first PDC region), the applied voltage is dropped mainly across the DBQW, and the electric field in the collector core is rather small, Fig. 7(a). Any optical loss increase with the applied voltage is mainly due to the thermal effects induced by the current flow, which rise linearly with the current. Above resonance (in the NDC and on the second PDC region), the additional applied bias voltage is dropped mainly across the depleted part of the collector spacer layer, Fig. 7(b), and the electric field magnitude is now much stronger than on the first PDC region, inducing large light absorption. The thermal optical absorption is now much less important because the current flowing through the devices biased on the valley region is significantly lower.

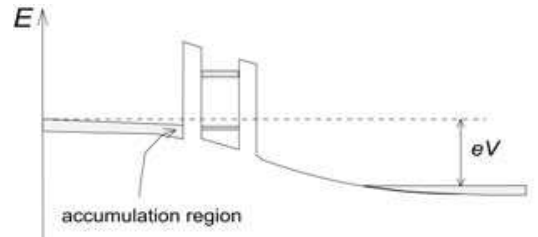

(a)

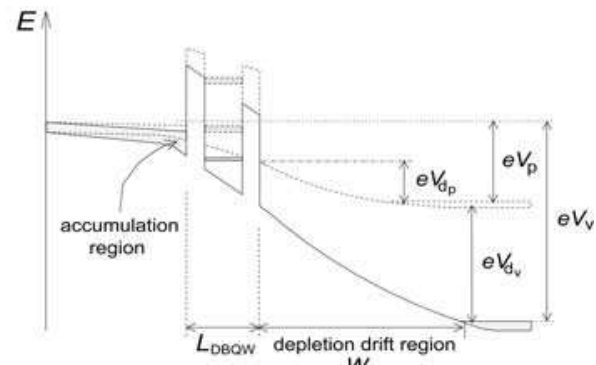

(b)

Fig. 7. Effect of applied biased on RTD-OW $\Gamma$-band: (a) before the peak and (b) on the valley. 
The electric field enhancement $\Delta E_{V P}$ induced by the peak to valley switching can be estimated as (Figueiredo, 2000)(Figueiredo et al., 2001):

$$
\Delta E_{V P} \simeq \Delta V_{V P} / W_{\text {dep }}+\left(W_{\text {dep }} / 2 \varepsilon v_{\text {sat }}\right) \Delta J_{P V}
$$

where $\Delta V_{V P}$ is the voltage dropped across the depletion region, $\Delta J_{P V}$ is the corresponding current density change, $v_{\text {sat }}$ is the carrier saturation velocity and $W_{\text {dep }}$ is the depletion thickness. At a given photon energy the absorption change induced by the electric field enhancement due to the peak to valley switching is given by (Figueiredo, 2000):

$$
\Delta \alpha\left(\hbar \omega, \Delta E_{V P}\right)=\alpha\left(\hbar \omega, E_{V}\right)-\alpha\left(\hbar \omega, E_{P}\right) \approx \alpha\left(\hbar \omega, \Delta E_{V P}\right)
$$

where $\alpha(\hbar \omega, E)$ is given, in the weak field approximation, by the Franz-Keldysh effect electroabsorption coefficient (Chuang, 1995)(Keldysh, 1958). The light modulation depth due to the peak to valley switching can be calculated using (Chuang, 1995):

$$
R_{V P}(\mathrm{~dB}) \approx 4.343 \gamma_{f} \Delta \alpha\left(\hbar \omega, \Delta E_{V P}\right) \ell
$$

where $\gamma_{f}$ is the optical filling factor which corresponds to the fraction of the optical power guided in the depleted region of the waveguide, and $\ell$ is the RTD-OW electrically active length, defined by the RTD metal contacts length [see Fig. 6(b)]. The measured FranzKeldysh effect effective band-edge shift to longer wavelengths can be compared with the value given by theory (Chuang, 1995)(Keldysh, 1958):

$$
\Delta \lambda_{g} \simeq\left(\lambda_{g}^{2} / h c\right)\left(e^{2} h^{2} / 8 \pi^{2} m_{r}\right)^{1 / 3} \Delta E_{V P}^{2 / 3}
$$

The measured $\Delta \lambda_{g}$ gives an independent way to determine the electric field change $\Delta E_{V P}$ induced by the peak to valley switching.

As mentioned, RTD-OWs designed to show considerable NDC with a significant portion of the waveguide core being depleted at bias voltages higher than the peak voltage can have their operation point switched between the two $I-V$ PDC regions by small high frequency ac signals $(<1 \mathrm{~V})$. This leads to high speed electric field switching, resulting in high frequency modulation of the waveguide optical transmission loss. In this mode of operation the RTD-OW is called a resonant tunnelling diode electro-absorption modulator (RTDEAM). In the RTD-EAM the modulation depth depends essentially on the overlap between the electric field in the collector depleted volume and the optical mode. The peak to valley electric field magnitude boost is determined mainly by the NDC region characteristics, $\Delta V$ and $\Delta J$. Figure 8 represents schematically the light absorption on the collector depleted region induced by the Franz-Keldysh effect when the RTD-OW is biased on the valley region, Fig. 8(a), and the change in the absorption coefficient associated with the bistable switching of the device plotted against wavelength, Fig. 8(b).

The device concept was implemented using AlGaAs ternary material system for operation on $900 \mathrm{~nm}$ optical window, and InGaAlAs quaternary compound to work on $1300 \mathrm{~nm}$ and $1550 \mathrm{~nm}$ optical windows, where the optical fibre present zero dispersion and have the lowest losses, respectively. For operation in the $900 \mathrm{~nm}$ spectral region, GaAs was used to form the waveguide core and the quantum well; AlAs and AlGaAs were employed to form the barriers and waveguide cladding layers, respectively. For operation at around $1550 \mathrm{~nm}$, 
(a)
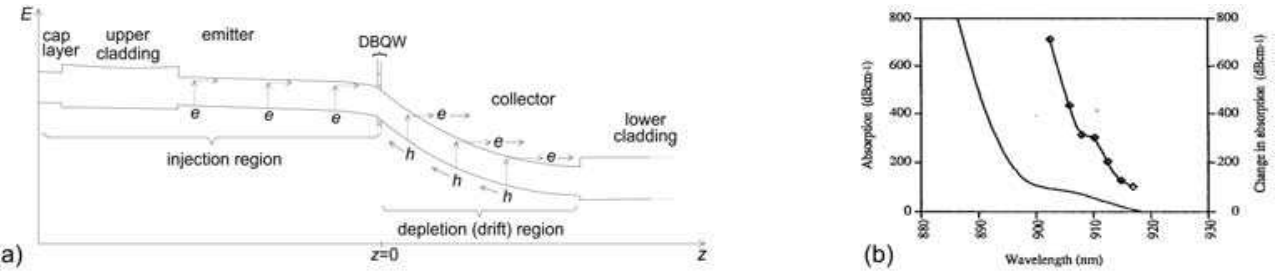

Fig. 8. (a) Schematic diagram of light absorption induced by Franz-Keldysh effect in a RTDOW biased around the valley point. (b) Change in absorption produced by the change in the voltage characteristic of the NDC pulse plotted with the absorption in $\mathrm{dB} / \mathrm{cm}$ of bulk GaAs against wavelength (McMeekin et al., 1994).

the InGaAlAs quaternary material system was used to implement the waveguide core and the quantum well, with $\mathrm{AlAs}$ and $\operatorname{In}_{0.48} \mathrm{Al}_{0.52} \mathrm{As} / \mathrm{InP}$ being employed for the barriers and the waveguide cladding layers, respectively. The InGaAsP quaternary compound also allows operation on $1300 \mathrm{~nm}$ and $1550 \mathrm{~nm}$ optical windows but was not used. A detailed description of the RTD-OW structures implemented can be found in (Figueiredo, 2000). Next we describe the experimental operation of RTD-OW electro-absorption modulators on the optical communication windows around $900 \mathrm{~nm}$ and $1550 \mathrm{~nm}$.

\subsection{RTD-OW operation as EAM at $900 \mathbf{~ n m}$}

The RTD-OW operation as an electro-absorption modulator at around $900 \mathrm{~nm}$ was achieved by growing the waveguide and DBQW layers using the AlGaAs/GaAs material system on semi-insulating GaAs. The GaAs waveguide core was made $1 \mu \mathrm{m}$ thick to allow easy end-fire light coupling, with $n$-type Si doping concentration of $2 \times 10^{16} \mathrm{~cm}^{-3}$; the cladding layers were made of $\mathrm{Al}_{0.33} \mathrm{Ga}_{0.67} \mathrm{As}$, a direct band-gap compound alloy, with $\mathrm{Si}$ doping concentration around $2 \times 10^{18} \mathrm{~cm}^{-3}$. The refractive index difference between the core and cladding layers around 0.224 at $900 \mathrm{~nm}$ is sufficiently to obtain efficient light confinement with relatively thin cladding layers. The upper cladding layer thickness was made $300 \mathrm{~nm}$ thick, twice the reciprocal of the optical waveguide first mode exponential decaying factor, to keep the device series resistance low. Because the waveguide core and the substrate have similar real refractive indices, the lower cladding layer was made $600 \mathrm{~nm}$ thick with Si doping concentration of $2 \times 10^{18} \mathrm{~cm}^{-3}$, to act as an isolation layer separating the core from the substrate, in order to significantly reduce radiation leakage into the GaAs substrate. The DBQW consisted of a $7 \mathrm{~nm}$ GaAs quantum well sandwiched between $1.4 \mathrm{~nm}$ AlAs barriers. The detailed description and fabrication of AlGaAs/GaAs structures can be found in (Figueiredo, 2000). Figure 9 shows the top view of a RTD-OW die and a packaged device.

When dc biased in the NDC region, all tested devices showed instabilities at around few MHz. These where removed connecting devices to the dc power supply via a wide bandwidth bias-T. In certain cases, a high frequency energy-storage element, such as a coax transmission line, was inserted between the RTD and the bias-T, resulting in a RTD-EAM transmission line relaxation oscillator whenever the cavity characteristic frequency was within the NDC bandwidth (Figueiredo et al., 1999). Typical electrical relaxation oscillations due to a $15 \mathrm{~cm}$ long coaxial transmission line are shown in Fig. 10(a). The relaxation oscillations RF spectra show harmonic components up to $15 \mathrm{GHz}$ (Figueiredo, 2000). The free-running oscillation frequency was changeable by varying the optical power coupled 

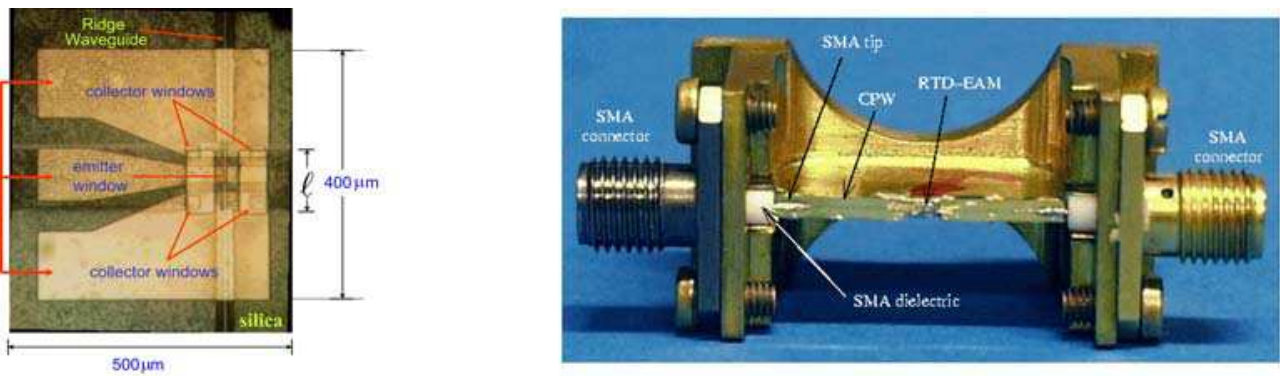

Fig. 9. RTD-OW die top view and a packaged device. The parameter $\ell$ here represents devices electrical active length, which with the waveguide width defines devices active area. into the RTD-EAM, as shown in Fig. 10(b); in the cases observed the free-running frequency decreased when the coupled optical power was increased. In a circuit with a free-running oscillation frequency around $470 \mathrm{MHz}$, a tuning range of $10 \mathrm{MHz}$ was observed. The frequency tuning effect is mainly due to the creation of charge carriers in the depletion region that reduces the device series resistance and moves the operating point through the NDC region, which change the device impedance [mainly the capacitance and the negative differential resistance (NDR)]. In the experiment light from a tunable Ti:sapphire laser emitting at around $900 \mathrm{~nm}$ was used; the optical power was kept to few $\mathrm{mW}$ in order to avoid damaging waveguide input facet.

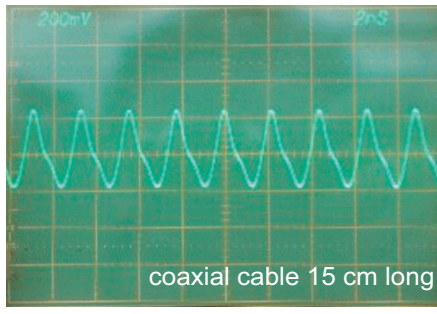

(a)

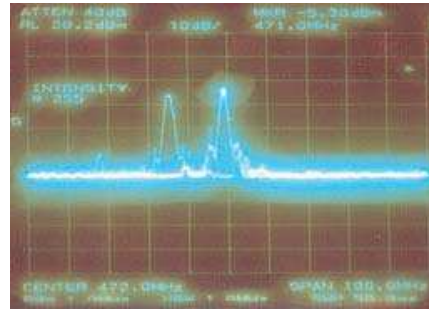

(b)

Fig. 10. (a) Self-sustained oscillations in a RTD-EAM connected via a $15 \mathrm{~cm}$ long coaxial line. (b) Self-oscillations frequency tuning induced by incident light.

The free-running relaxation oscillation frequency is also affected by the dc bias voltage because of the device intrinsic impedance dependence on the voltage. These behaviours can be used to implement both optical controlled oscillators (OCOs) and voltage controlled oscillators (VCOs). The OCO can be used to optically control microwave oscillators, and will be briefly analyzed when discussing the RTD-OW operation as photo-detector. The VCO behaviour makes possible operating the RTD-EAM as an optoelectronic voltage controlled oscillator (OVCO) since the electric field across the depleted collector region also selfoscillates at the free-running frequency, self-modulating the transmission properties of the waveguide. Before discussing OVCO operation we present electro-absorption response of the RTD-EAM. The RTD-EAM waveguide transmission spectra at zero bias, at slightly below the peak, and just above the valley points, are shown in Fig. 11(a) for devices with active areas around $800 \mu \mathrm{m}^{2}$. (The devices were not dc biased in the NDC region in order to avoid self-oscillation.) As the applied voltage increases from the peak to the valley point, 
there is a sharp drop in the waveguide transmission at wavelengths in the range $890 \mathrm{~nm}$ to $910 \mathrm{~nm}$. The observed Franz-Keldysh absorption band-edge shift was around $12 \mathrm{~nm}$ which compares to $9 \mathrm{~nm}$ estimated using equation 12, taking in consideration the approximations made (Figueiredo, 2000). Figure 11(b) presents the optical modulation depth as a function of the operating wavelength due to the transition between the two positive PDC regions induced by a square signal with peak-to-peak voltage slight higher than $\Delta V_{V P}=V_{V}-V_{P}$. Modulation depth up to $13 \mathrm{~dB}$ around $908 \mathrm{~nm}$ was achieved. Modulation depths up to $18 \mathrm{~dB}$ were observed in waveguides with $400 \mu \mathrm{m}$ active length and $4 \mu \mathrm{m}$ wide ridges.

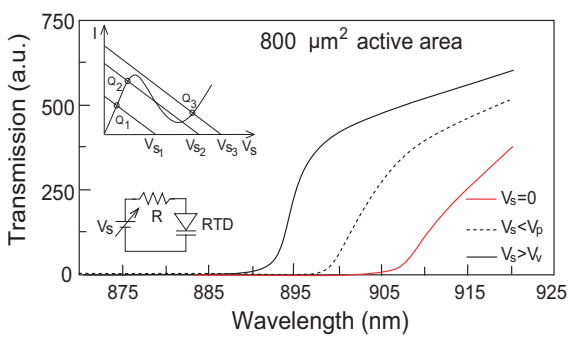

(a)

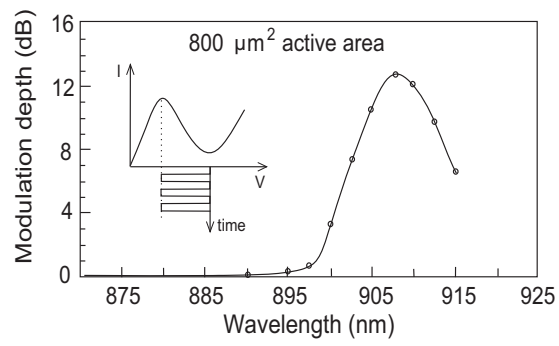

(b)

Fig. 11. (a) AlGaAs RTD-EAM optical transmission spectrum at zero volts, around the peak and at the valley region. (b) Modulation depth as function of the operating wavelength due to peak-to-valley switching induced by a square voltage waveform.

Direct modulation was obtained dc biasing the RTD-EAM slightly above the valley point and injecting through a wide band bias-T the rf modulating signals. Figure 12 shows examples of modulation due to $950 \mathrm{MHz}$ and $16 \mathrm{GHz}$ rf signal voltages. In both cases the driving signals amplitude was kept slightly larger than $\Delta V_{V P} \sim 0.4 \mathrm{~V}$. Optical modulation depths as high as $11 \mathrm{~dB}$ were achieved (Figueiredo et al., 1999)(Figueiredo, 2000). The 16 $\mathrm{GHz}$ response shown in Fig. 12(b) gives a good estimation of the bandwidth and modulation depth potential of the devices. The modulation efficiency characterized by the bandwidth-to-drive-voltage ratio, defined as the ratio of the operation bandwidth to the operating voltage for at least $10 \mathrm{~dB}$ modulation depth, was $40 \mathrm{GHz} / \mathrm{V}$ (Figueiredo et al., 1999)(Figueiredo, 2000).

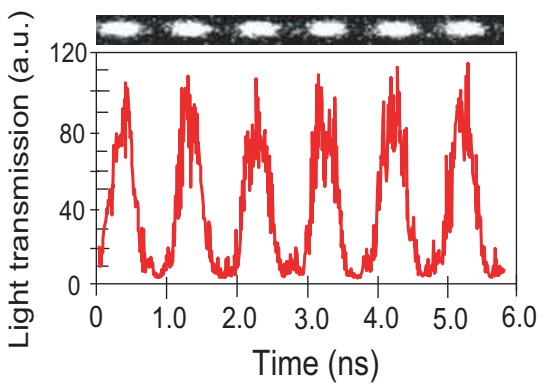

(a)

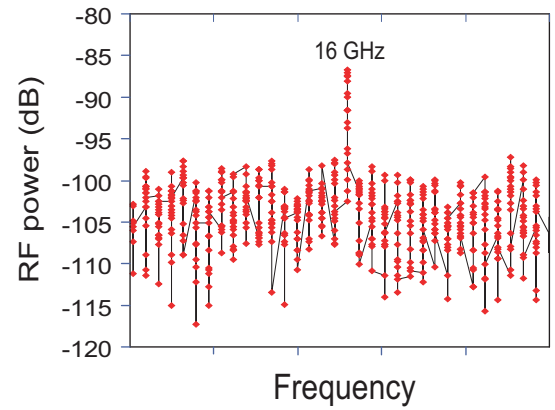

(b)

Fig. 12. (a) Direct modulation at around $950 \mathrm{MHz}$, with modulation depth up to $11 \mathrm{~dB}$ ( $\lambda=908 \mathrm{~nm}$ ). (b) Modulator response to a $16 \mathrm{GHz}$ rf signal. 
As discussed previously, when dc biased in the NDC region and connected to a bias-T through a coaxial line the RTD-EAM can operate in the self-oscillation mode, producing an optical output modulated by the NDC induced relaxation oscillations, at frequencies determined by the electrical length of the transmission line, as shown in Fig. 13.

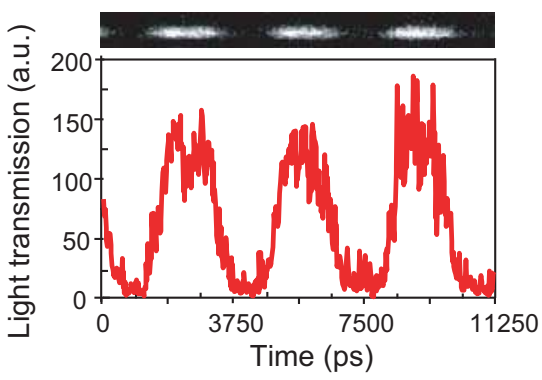

(a)

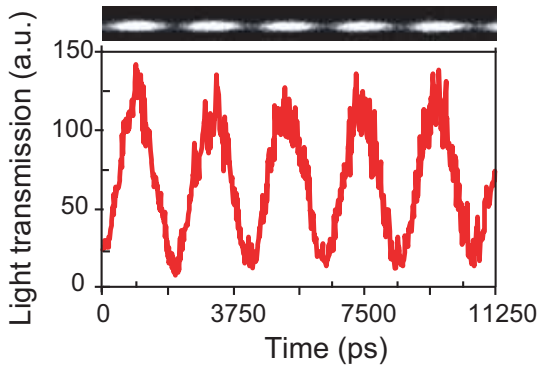

(b)

Fig. 13. Optical responses measured with Streak Camera of RTD-EAM transmission line relaxation oscillators with lines $15 \mathrm{~cm}$ (a) and $10 \mathrm{~cm}$ (b) long.

The AlGaAs RTD-EAM operation modes discussed above can be employed in LANs systems primarily as devices for electrically controlling guided-wave optical signals in the $880 \mathrm{~nm}$ to $1100 \mathrm{~nm}$ wavelength range such as waveguide intensity modulators, directional couplers and optical switches. The capability to operate in relaxation oscillation mode can be applied in clock extraction circuits, for optical pulse generation and de-multiplexing in optical time division multiplexed systems.

\subsection{RTD-OW operation as EAM at $1550 \mathrm{~nm}$}

The AlGaAs/GaAs RTD-EAM achieved performances led the work to the demonstration of device concept operation at $1550 \mathrm{~nm}$, where standard single-mode optical fibres have lowest losses (Liu, 1996). For band gap energies between $0.75 \mathrm{eV}$ and $1.439 \mathrm{eV}$, quaternary alloys lattice matched to $\mathrm{InP}$, which combine $\mathrm{In}, \mathrm{Ga}, \mathrm{Al}$, and $\mathrm{As}\left(\mathrm{In}_{1-x-y} \mathrm{Ga}_{x} \mathrm{Al}_{y} \mathrm{As}\right)$ or $\mathrm{In}, \mathrm{Ga}, \mathrm{As}$, and $\mathrm{P}\left(\mathrm{In}_{1-x-y} \mathrm{Ga}_{x} \mathrm{As}_{1-y} \mathrm{P}_{y}\right)$, can be used (Chuang, 1995)(Figueiredo, 2000). The RTD-OW concept operating at $1550 \mathrm{~nm}$ was demonstrated using InGaAlAs lattice matched to InP because phosphorus based heterostructures have lower conduction band discontinuity, which prevents strong localization of electrons in the lower band gap material. Moreover, they are difficult to grow with conventional MBE systems due to the need to handle solid phosphorus and high concentration of phosphorus at its vapour pressure, and also due to the difficulty to control As/P ratio. ${ }^{1}$ The InGaAlAs material system shows more favorable material properties such as higher electron mobility, lower effective mass and superior conduction bandedge discontinuity (Figueiredo, 2000). As a consequence it is expected the InGaAlAs RTD-OW shows superior speed and modulation depth performance mainly to the InGaAs RTD higher peak current density and peak-to-valley current ratio, and smaller operating voltage. The In- GaAlAs quaternary system lattice matched to InP allows as well operation at $1300 \mathrm{~nm}$, where standard single-mode optical fibres show zero dispersion (Chuang, 1995)(Figueiredo, 2000).

1 Structures incorporating InGaAsP are usually grown by MOCVD (Bohrer et al., 1993). 
The InGaAlAs RTD-OW schematic wafer structure for operation at $1550 \mathrm{~nm}$ is shown in Fig. 14, with wafer $\Gamma$-valley and refractive index profiles. The core consisted of two $\operatorname{In}_{0.53} \mathrm{Ga}_{0.42} \mathrm{Al}_{0.05} \mathrm{As}$ layers (refractive index of 3.56), $0.5 \mu \mathrm{m}$ thick each, with a band-gap energy around $0.826 \mathrm{eV}$ (absorption band-edge wavelength around $1500 \mathrm{~nm}$ ), to allow operation at $1550 \mathrm{~nm}$ when biased around the peak voltage. The upper cladding was implemented using a layer of $\mathrm{In}_{0.52} \mathrm{Al}_{0.48} \mathrm{As}$, refractive index of 3.24. Because $\mathrm{InP}$ refractive index at $1550 \mathrm{~nm}$ ( 3.17) is considerably smaller than the $\operatorname{In}_{0.53} \mathrm{Ga}_{0.42} \mathrm{Al}_{0.05} \mathrm{As}$ refractive index, the $n$-type InP substrate acted as lower cladding region. As previously discussed, the upper cladding layer thickness was made $300 \mathrm{~nm}$ thick. A detailed description of the wafer structure is given in (Figueiredo, 2000). Most of the RTD-EAMs characterized were $4 \mu \mathrm{m}$ wide ridges with $200 \mu \mathrm{m}$ active lengths. Typical current-voltage characteristic of $4 \mu \mathrm{m} \times 200$ $\mu \mathrm{m}$ InGaAlAs/InP RTD-EAM is presented in Fig. 5(b) (section 2), showing PVCR around 3. These devices showed valley-to-peak voltage differences $\Delta V_{V P} \sim 0.8 \mathrm{~V}$, with peak-to-valley current density differences $\Delta J_{P V} \sim 10 \mathrm{kA} / \mathrm{cm}^{2}$. (Typical GaAs/AlAs devices show $\Delta V_{V P} \sim 0.4$ $\mathrm{V}$ and $\Delta J_{P V} \sim 5 \mathrm{kA} / \mathrm{cm}^{2}$.)

\begin{tabular}{|c|c|}
\hline $\mathrm{In}_{0.52} \mathrm{Ga}_{0.48}$ As contact layer & $2 \times 10^{19} \mathrm{~cm}^{-3}$ \\
\hline $\mathrm{In}_{0.52} \mathrm{Al}_{0.48}$ As upper cladding & $2 \times 10^{18} \mathrm{~cm}^{-3}$ \\
\hline $\begin{aligned} & \ln _{0.53} \mathrm{Ga}_{0.42} \mathrm{Al}_{0.05} \text { As upper core } \\
= & \mathrm{DBQW}=\end{aligned}$ & $5 \times 10^{16} \mathrm{~cm}^{-3}$ \\
\hline $\ln _{0.53} \mathrm{Ga}_{0.42} \mathrm{Al}_{0.05}$ As lower core & $5 \times 10^{16} \mathrm{~cm}^{-3}$ \\
\hline $\mathrm{n}+\mathrm{InP}$ substrate lower cladding & \\
\hline
\end{tabular}

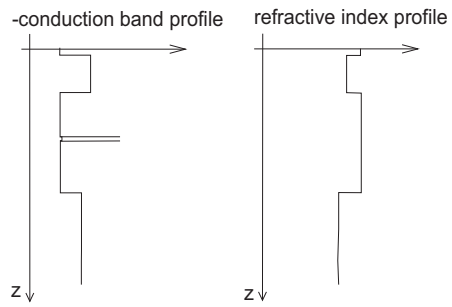

Fig. 14. InGaAlAs RTD-EAM structure, $\Gamma$-valley and refractive index profiles.

The devices' frequency response was investigated by on wafer impedance measurements in the $45 \mathrm{MHz}$ to $18 \mathrm{GHz}$ frequency range for all values of bias voltage. The results indicate the InGaAlAs RTD-EAM small signal equivalent circuit consists of a capacitance $C$ in parallel with a non-linear resistor $R_{d}(V)$, in series with a resistance $R_{S}$; the series inductance was found to be negligible (Figueiredo, 2000)(Alkeev et al., 2000). The devices average capacitance $C$ and shunt resistance $R$ around the NDC region were $1 \mathrm{pF}$ and $-15 \Omega$, respectively; the $R_{S}$ was typical few ohms (less than $5 \Omega$ ) ((Figueiredo, 2000)(Alkeev et al., 2000). The device switching time can be estimated as $t_{R} \simeq 4\left(\Delta V_{V P} / \Delta J_{P V}\right) C_{V}$, where $C_{V}$ is the device capacitance per unit area $\left(C_{V} \approx \varepsilon / W_{\text {dep }}(V)\right)$. For the devices tested the expected modulation bandwidth was superior to $30 \mathrm{GHz}$ (Figueiredo, 2000).

Following the frequency characterization, the waveguide low frequency electro-absorption response was characterized with no applied voltage, dc biased at slightly below the peak voltage, and on the valley region; the device was not dc biased in the NDC region in order to avoid self-oscillations. Light from a Tunics diode laser, tunable in the wavelength range $1480 \mathrm{~nm}$ to $1580 \mathrm{~nm}$ was fibre coupled to the waveguide, with the light output fibre coupled to an optical power meter or a high bandwidth photo-detector. The InGaAlAs/InP RTDEAM waveguide transmission spectrum change due to the Franz-Keldysh effect absorption bandedge broadening induced by peak-to-valley switching is indicated in Fig. 15(a). The measured wavelength band-edge shift was $43 \mathrm{~nm}$, which compares quite well with the estimation of $46 \mathrm{~nm}$, equation 12. The low frequency electro-absorption response showed 5 
$\mathrm{dB}$ absorption changes induced by $1 \mathrm{mV}$ dc voltage increments, an exceptionally high transmission change per unit of voltage (Figueiredo, 2000). Figure 15(b) shows modulator response as function of the dc bias voltage when driven by $3 \mathrm{GHz}$ voltage signals of amplitude from $1 \mathrm{mV}$ to $100 \mathrm{mV}$; also represented is the RTD-EAM dc $I-V$ characteristic. The rf photo-detected power increased by about $15 \mathrm{~dB}$ when the device dc bias point moved from the peak to the valley region at driving amplitudes as low as $50 \mathrm{mV}$. An indication the modulator can be driven by very low voltage signals due to its intrinsic built-in electrical amplifier.

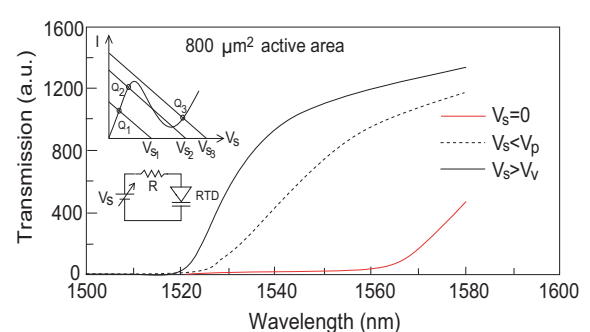

(a)

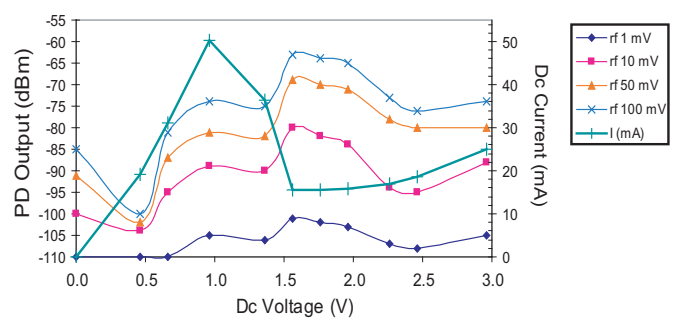

(b)

Fig. 15. (a) InGaAlAs RTD-EAM transmission spectrum in the wavelength range $1500 \mathrm{~nm}$ to $1580 \mathrm{~nm}$, with the applied voltage as a parameter. (b) Modulator response as function of the dc bias voltage when driven by $3 \mathrm{GHz}$ rf signals, with injected amplitude as a parameter.

RTD-EAM high frequency optical characterisation employed a microwave synthesized signal generator with a maximum output of $+20 \mathrm{dBm}$ and an upper frequency limit of 26 $\mathrm{GHz}$ (Figueiredo, 2000). Figure 16(a) shows the modulation depth as function of the light wavelength induced by the transition between the two PDC regions produced by a square signal with peak-to-peak voltage slight higher than $\Delta V_{V P} \sim 0.8 \mathrm{~V}$. The devices were dc biased in the valley region in order to minimize thermal effects and avoid self-oscillations. Modulation depths up to $28 \mathrm{~dB}$ were measured on devices with active areas around 800 $\mu \mathrm{m}^{2}$, more than $10 \mathrm{~dB}$ superior to the values observed on the AlGaAs/GaAs devices. The modulator response up to $26 \mathrm{GHz}$ driving signals for two power values is shown in Fig. 16(b).

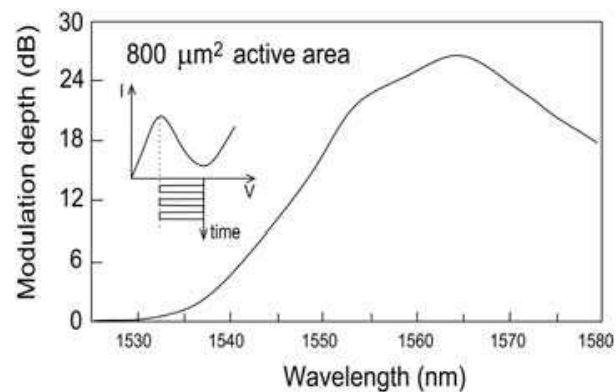

(a)

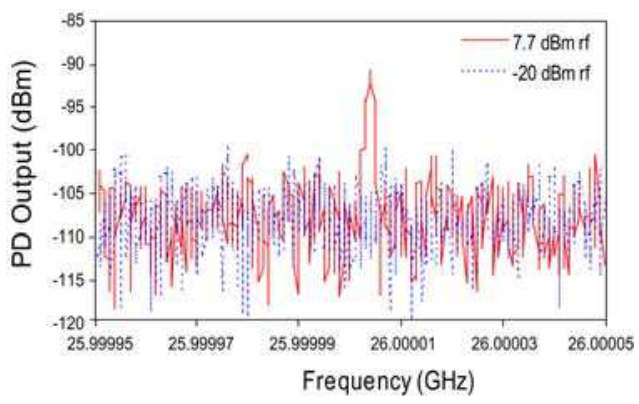

(b)

Fig. 16. (a) Modulation depth as function of the wavelength. (b) Spectrum of the $26 \mathrm{GHz}$ photo-detected signal at the modulator driving power of $-20 \mathrm{dBm}$ and $+7.7 \mathrm{dBm}$. 
The photo-detected power increases more than $10 \mathrm{~dB}$ when the driving rf power rises from $20 \mathrm{dBm}$ to $+7.7 \mathrm{dBm}$, an indication the device is capable to achieve modulation extinction ratios higher than $10 \mathrm{~dB}$ induced by low power driving signals, less than $10 \mathrm{~mW}$, as the consequence of the built-in electrical amplifier. The RTD intrinsic amplifier effect reduces substantially the rf power required for modulation. This on-chip amplification can eliminate the need of an external $\mathrm{rf}$ amplifier which is usually required to drive EAMs (Wakita et al., 1998).

\subsection{RTD-OW operation as photo-detector at $1550 \mathrm{~nm}$}

Light-wave receivers contain photo-detecting devices that convert the light-wave carrier modulation into an electrical signal that needs to be amplified before processing to recover the information signal (Liu, 1996)(Einarsson, 1996). The amplifying circuitry can be the system main penalty in terms of cost and power. We are currently investigating a receiver based on the RTD-OW to take advantage of the RTD intrinsic built-in amplifier.

Because in the RTD-OW the light interaction length is much longer than in conventional RTDs, the RTD-OW will produce substantial inter-band absorption, giving rise to a responsivitygain superior to the one obtained with conventional photo-detectors (Moise et al., 1995). The RTD-OW photo-detection characterization employed light from a Tunics tunable laser diode capable to be directly modulated up to $1 \mathrm{GHz}$ and operate in the mode locked regime at $5 \mathrm{GHz}$. Figure 17(a) presents the rf power capture level when light modulated at $1 \mathrm{GHz}$ was end-fire coupled to the waveguide. The RTD-OW responsitivitygain increases with the transition from peak to valley voltage, $V_{p}$ and $V_{v}$, by more than 15 dB. Figure 17(b) shows the photo-detected rf power as function of wavelength for dc bias on the peak and on the valley. Photo-detection of mode locked light at $5 \mathrm{GHz}$ showed similar performance.

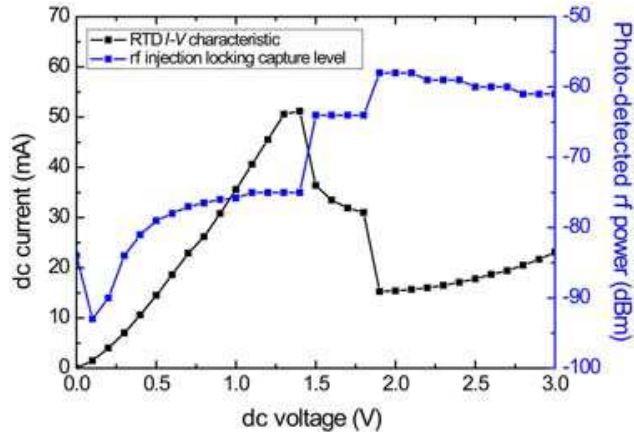

(a)

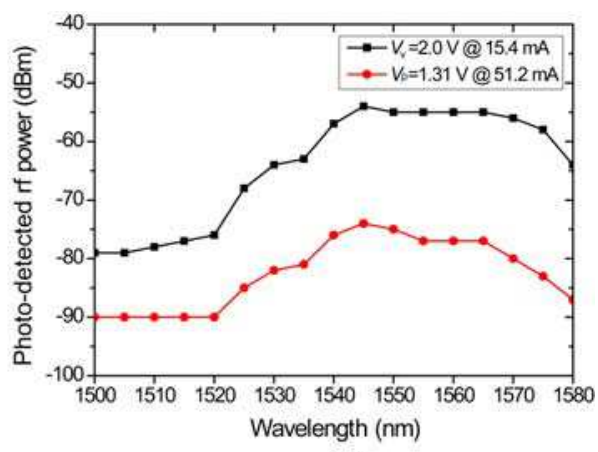

(b)

Fig. 17. (a) RTD-OW I - V characteristic and rf power produced due $1550 \mathrm{~nm}$ optical signals modulated at $1 \mathrm{GHz}$. (b) Rf power produced optical signals modulated at $1 \mathrm{GHz}$ as function of wavelength, at DC biased on the peak and on the valley.

When dc biased in the NDC region the RTD-OW self-oscillations lock to the injected light subcarrier, producing electrical signals that emulate the optical subcarrier. We are currently investigating the synchronization between optical subcarriers and RTD-OW free-running oscillations to transfer the information bearing signals such as Phase Shifted Keyed signals from the optical to the rf wireless domain without the need of an external amplifier (Romeira ${ }^{a}$ et al., 2009). 


\section{RTD laser diode integration}

A light-wave transmitter comprises a driving circuit and a LED or a laser diode which converts the supplied electrical signal containing the information into a light-wave signal. Novel alternatives to traditional laser diode transistor-driver circuits have been proposed based on the integration of a DBQW with semiconductor light sources, since the DBQW layers fit well with the epitaxial layers that make up semiconductor light sources. Furthermore, since the RTD can act as a voltage controlled switch, low voltage digital signals can be employed to switch the RTD between on and off states. It is expected the light sources high-speed modulation characteristics will improve significantly. In what follows we make a brief description of the first monolithic integration of a RTD with an optical communication laser operating at $1500 \mathrm{~nm}$, and give a detailed report on recent advances on the hybrid integrated version operating at $1550 \mathrm{~nm}$ optical windows.

\subsection{RTD-LD monolithic integration}

The first integration of a DBQW-RTD and an optical communication laser operating at around $1500 \mathrm{~nm}$ was reported by (Slight \& Ironside, 2007). The device consisted of a vertical integration of a DBQW on an InGaAs/InGaAlAs multiple quantum well laser structure. Such integration is straightforward as the RTD section requires only the growth of four to six extra epilayers above a laser structure grown on $p$-type InP substrate, allowing the RTD to be implemented on the laser junction $n$-type region. The DBQW was made of a $5 \mathrm{~nm}$ InGaAs well and $2 \mathrm{~nm}$ AlAs barriers. The devices fabricated were ridge waveguides with the DBQW situated in the ridge between the laser section and the $n$-type contact, Fig. 18(a). A detailed description of device structure and fabrication can be found in (Slight et al., 2006). The RTD-LD current-voltage characteristic emulates the RTD non-linear $I$ - $V$ curve, hysteresis and bistability (Slight \& Ironside, 2007). Figure 18(b) shows a typical RTD-LD optical-voltage characteristic at $130 \mathrm{~K}$, where a hysteresis window is clearly seen; bistable operation was also observed (Slight et al., 2006). The results demonstrate the feasibility of monolithically integrated RTDs with LDs. In order to achieve room temperature operation a new wafer was designed and device fabrication will start soon. Further investigation of the monolithic RTD-LD will include high-frequency operation characterization.

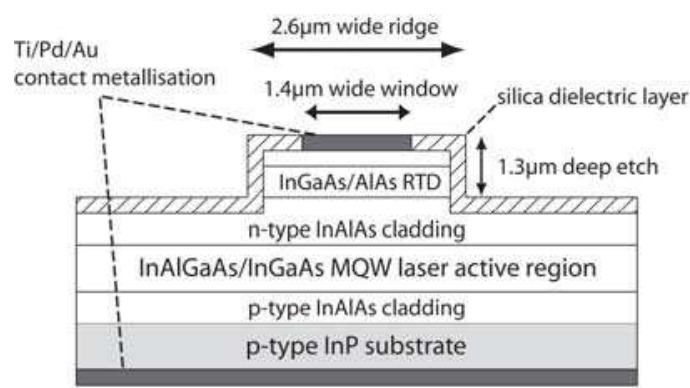

(a)

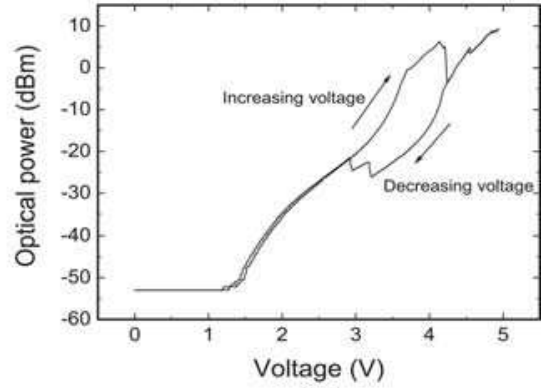

(b)

Fig. 18. (a) Cross section schematic of the ridge waveguide RTD-LD. (b) optical-voltage $(P-V)$ characteristic at $130 \mathrm{~K}$, clearly showing bistability and hysteresis. 


\subsection{RTD-LD hybrid circuit}

Once demonstrated the bistable operation of monolithically integrated RTD-LDs the work concentrated on the hybrid integrated circuit (HIC) versions using components similar to the targeted monolithic integrated device. Although without the monolithic expected superior performance, laboratory hybrid RTD-LDs are easy and much less costly to implement, allowing to study both components behaviour separately. The first HICs combined an InGaAs RTD and a commercial prototype laser diode (Slight \& Ironside, 2007). The In- GaAs RTD used was fabricated from RTD epi-material originally used in the work described in section 3; the laser diode was a $5 \mu \mathrm{m}$ ridge wide waveguide designed for continuous-wave (CW) emission at around $980 \mathrm{~nm}$. The RTD and LD were attached to a small copper block using electrically conductive silver epoxy resin, and connected in series through $25 \mu \mathrm{m}$ diameter gold wire bonding, as schematically represented in Fig. 19(a). Also shown are LD and RTD-LD experimental and PSPICE simulated $I-V$ characteristics, Fig. 19(b) (Slight \& Ironside, 2007). The PSPICE code used can be found in (Slight \& Ironside, 2007).

The RTD reduces significantly the laser driving circuits' complexity by taking advantage of its high nonlinear $I-V$ characteristic, with the NDC region providing electrical gain to the circuit. The RTD features make possible to operate the RTD-LD as an autonomous OVCO, where the running frequency is fine tuned by the dc bias voltage. Light modulation due to relaxation oscillations at $5 \mathrm{MHz}$ was observed with optical power on/off or extinction ratio up to $31 \mathrm{~dB}$. Moreover, because of RTD bistability the RTD-LD optical output is also bistable, as shown in Fig. 19(c), a feature of particularly convenience for non-return to zero (NZR) digital modulation.

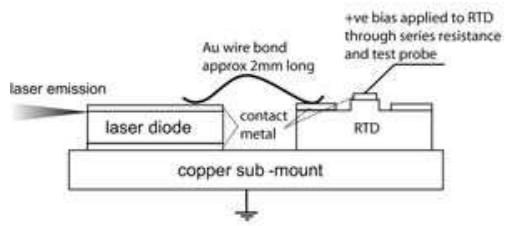

(a)

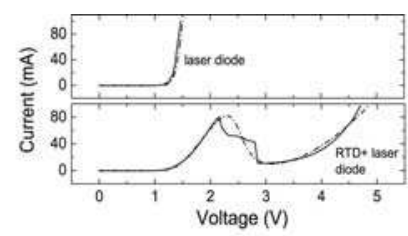

(b)

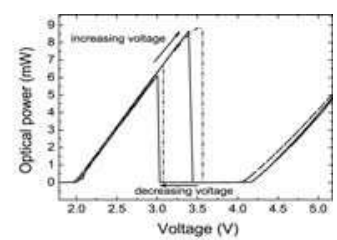

(c)

Fig. 19. (a) Illustration of the RTD-LD module. (b) LD and RTD-LD I - V characteristics. (c) Optical power versus voltage $(P-V)$ characteristic showing bistability and a $410 \mathrm{mV}$ wide hysteresis loop. Dashed lines show the PSPICE simulations.

To increase the relaxation oscillations free-running frequency the hybrid circuit was redesigned. InGaAlAs RTD-OW devices with areas around $1000 \mu \mathrm{m}^{2}$ were used together with commercial prototype ridge waveguide laser dies designed for $\mathrm{CW}$ operation with emission at around $1550 \mathrm{~nm}$ with $5 \mathrm{~mW}$ average output power, bandwidth of $20 \mathrm{GHz}$ and threshold current $I_{\text {th }}$ around $6 \mathrm{~mA}$. The new circuits layouts were mounted directly onto the surface of printed circuit boards (PCBs) containing a $50 \Omega$ copper microstrip transmission line laminated onto the non-conductive PCB substrate. These new improvements on the hybrid RTD-LD circuits lead to some significant breakthroughs: (i) the use of commercial communications laser diodes operating at $1550 \mathrm{~nm}$; (ii) the oscillation frequency went up to for more than two orders of magnitude by solving the instabilities associated to the dc bias circuitry; (iii) demonstration of operation as an autonomous relaxation oscillator in the $\mathrm{GHz}$ range, controlled by voltage; (iv) observation of new operation capabilities induced by injected periodic and phase modulated signals. 
In the improved circuits the RTD and LD components were attached directly onto the PCBs using silver epoxy resin and bond wires where used to connect the RTD emitter contact to LD, and the RTD collector contact to the $50 \Omega$ copper microstrip line, as shown in Fig. 20(a). A parallel resistor-capacitor shunt was incorporated as close as possible to the RTD-LD components to reduce the spurious oscillations and to act as a short circuit for the rf signals generated by the RTD-LD. The circuit shunt component values were typically $5 \Omega$ and 3.3 $\mathrm{nF}$. The dc bias and rf injected signals were applied via a wideband bias-T through the resistor-capacitor shunt that also acts as the circuit input port. The circuit electrical output port was defined by the PCB ground plane and the microstrip line, and corresponds to the RTD-LD series terminals as shown in Fig. 20(a). The laser optical output was coupled to a lensed fibre before photo-detection. The light coupling efficiency was estimated from the laser mode profile and single mode fiber characteristics to be around 10 per cent. In Fig. 20(b) are presented the typical $I-V$ characteristics of the LD (with the threshold current inset) and of two RTD-LD circuits, I and II, measured without the shunt resistorcapacitor. RTD-LD circuits I and II analysed here have similar PCB layout designs and LD and shunt components. The RTDs used in circuit I and II have approximately the same current peaks, $I_{p}$, but different valley currents, $I_{v}$, and thus different peak-to-valley current ratios. RTD-LD II was designed to have a lower bond wire length connection between RTD and LD components, which increased its oscillation frequency operation, as discussed below. In both cases $I_{t h}<I_{v}$, which meant that when dc biased in the NDC region, the lasers were working well above threshold current.

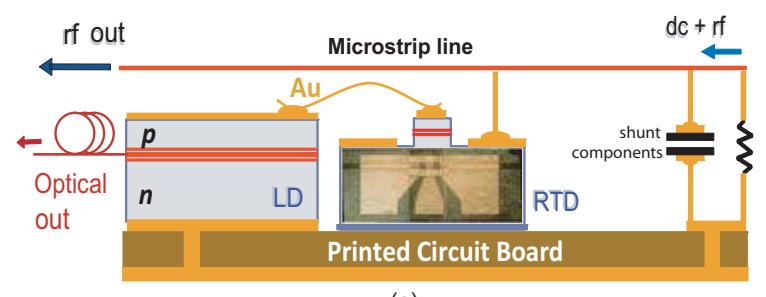

(a)

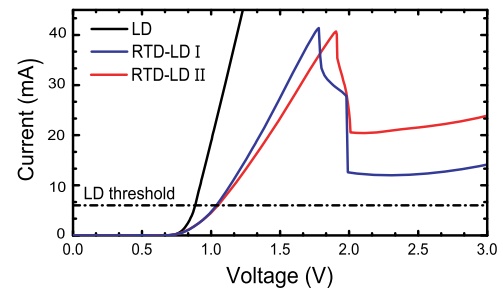

(b)

Fig. 20. (a) Layout of the improved hybrid RTD-LD circuit. (b) Current-voltage characteristic of the laser diode and two RTD-LD circuits, showing the RTD NDC is preserved by the RTDLD module.

The RTD-LD circuit of Fig. 20(a) can be represented by circuit electrical layout of Fig. 21(a). When dc biased in or close to the NDC region the laser diode is operating well above the threshold current the laser is well represented simple by its differential resistance. Because its capacitance is much larger than the RTD's, the RTD-LD module equivalent capacitance corresponded to the RTD intrinsic capacitance. This approximation seems reasonable since changing the laser diode did not alter the circuit free-running frequency whenever the lengths of the bond wires used to connect the RTD to the LD were identical. Indeed, the circuit of Fig. 21(a) behaves at rf frequencies like an $R L$ circuit connected to the RTD small signal equivalent circuit (a voltage dependent current source $F(V)$ in parallel with the RTDLD capacitance, as discussed in section 2.2). Its electric behaviour under external perturbation can be studied numerically using the small signal equivalent circuit shown in Fig. 21(b). The lumped LCR components of Fig. 21(b) represents the microstrip transmission line and wire bond equivalent inductance, the RTD intrinsic capacitance and the devices equivalent series resistance, respectively. 


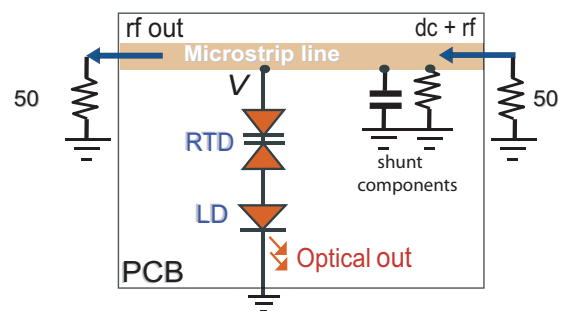

(a)

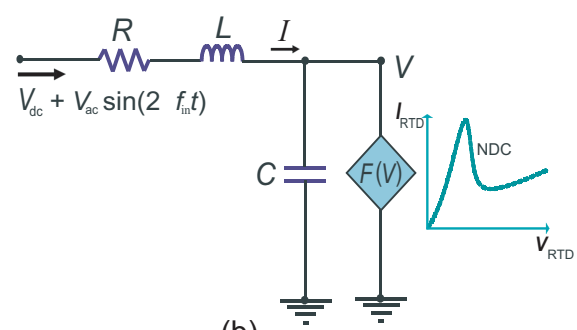

(b)

Fig. 21. (a) Electrical schematic of the RTD-LD circuit where $V$ represents the electrical output taken across the RTD-LD. (b) RTD-LD small-signal equivalent lumped circuit. $V_{a c}$ $\sin \left(2 \pi f_{\text {in }} t\right)$ represents an ac injected driving signal.

The maximum operating free-running frequency of circuit RTD-LD I was around $640 \mathrm{MHz}$, whereas for RTD-LD II the maximum observed free-running frequency was $2.15 \mathrm{GHz}$ (the maximum obtained with the hybrid circuits presented here). The RTD-LD II higher running frequency was mainly due to the smaller inductance achieved with this circuit layout due to the shortening of bond wires length used to connect the RTD to the LD, roughly from $5 \mathrm{~mm}$ to less than $2 \mathrm{~mm}$ that corresponded to a reduction of the equivalent inductance value from approximately $8 \mathrm{nH}$ to around $1.5 \mathrm{nH}$. In both circuits the estimated capacitance $C$ was $3 \mathrm{pF}$. These values when used in the electrical circuit model, Eq. 8, lead to theoretical maximum relaxation oscillation frequencies, given by $1 / 2 \pi \sqrt{L C}$, around $1.03 \mathrm{GHz}$ and $2.37 \mathrm{GHz}$, respectively.

\subsection{RTD-LD optoelectronic model}

When dc biased in the NDC region, the circuit of Fig. 20(a) behaves as a classic negativeresistance oscillator (Van der Pol, 1927). Since the circuit of Fig. 21(b) is similar to the circuit of Fig 4, apart from the injected ac driving signal $V_{a c} \sin \left(2 \pi f_{i n} t\right)$, we applied the same procedure, obtaining a second-order differential equation (see section 2.2), commonly referred as one of the generalized forced nonlinear Liénard systems (Romeira et al., 2008)(Figueiredo, 1970):

$$
\ddot{V}+H(V) \dot{V}+G(V)=V_{a c} \sin \left(2 \pi f_{i n} t\right)
$$

where $G(V)$ is a nonlinear force and $H(V) \dot{V}$ is the damping factor (see section 2.2).

To describe the RTD-LD optoelectronic behaviour we coupled equation 13 to the laser diode single mode rate equations that governs the interrelationship between carrier density and photon density. Assuming the laser oscillates in a single mode and the population inversion is homogeneous, the laser rate equations for photon density $S$ and injected carrier density $N$ are:

$$
\begin{aligned}
& \dot{N}=\frac{I}{q \vartheta}-\frac{N}{\tau_{n}}-g_{0}\left(N-N_{0}\right) \frac{S}{1+\varepsilon S} \\
& \dot{S}=g_{0}\left(N-N_{0}\right) \frac{S}{1+\varepsilon S}+\frac{S}{\tau_{p}}+\beta \frac{N}{\tau_{n}}
\end{aligned}
$$


where $I$ is the total current through the laser diode given by generalized Liénard's system, Eq. 13, plus the dc bias current; $q$ is the electron charge, $\vartheta$ is the laser active region volume, $\tau_{n}$ and $\tau_{p}$ are the spontaneous electron and photon lifetimes, respectively; $\beta$ is the spontaneous emission factor; $g_{0}$ is the gain coefficient; $N_{0}$ is the minimum electron density required to obtain a positive gain and $\varepsilon$ is the value for the nonlinear gain compression factor. The numerical analysis employed typical parameters of semiconductor laser diodes, as described in (Slight et al., 2008)(Romeira et al., 2008). The coupled system of equations 1315 has been successfully used to predict the experimental behaviour of RTD-LD electrical and optical outputs.

\subsection{RTD-LD optoelectronic voltage controlled oscillator}

It is well known that a single-port device that has a negative differential conductance in a portion of its operating range may be used as the basis of a bistable or multistable circuit, and can also be used to form astable circuits (relaxation oscillators), monostable circuits (singlepulse generators), and sine-wave generators (Brown et al., 1997). A simple way to implement a RTD oscillator is to couple a RTD dc biased in the NDC to a resonant tank circuit or a resonant cavity that provides frequency stability (the coupling location in the cavity can serve to partially match its impedance to that of the RTD). Such oscillator corresponds to a relaxation oscillator system since it operates by sequential transitions between unstable states. The RTDLD circuit of Fig. 20(a), whose circuit schematic is represented in Fig. 21 with the small signal equivalent circuit, operates as a relaxation oscillator when dc biased in the NDC region. The circuit free-running frequency is determined primarily by the round trip time of the ac feedback loop (effective length of equivalent transmission line from the shunt resistorcapacitor to the RTDLD module), in combination with the RTD and the LD parasitics (mainly the inductance from the wire bonding).

The RTD successive switching events (relaxation oscillations) produce sharp current pulses that modulate the laser output yielding sharp optical pulses at the relaxation oscillation fundamental frequency (free-running frequency). Typical RTD-LD self-sustained oscillation voltage output and photodetect optical waveforms are shown in Fig. 22. Figure 22(a) shows RTD-LD I voltage output waveform at free-running frequency around $600 \mathrm{MHz}$; Fig. 22(b) presents the photo-detected laser optical output modulated by the current relaxation oscillations with an on/off superior to $20 \mathrm{~dB}$.

The pulsed nature of the photo-detected laser optical output shown in Fig. 22 confirms the capacitive character of the current induced by the RTD switching (described in detail in (Brown et al., 1997)). The full width at half maximum (FWHM) of the photo-detected pulses is approximately 200 ps but this measurement is limited by the temporal acquisition resolution of the oscilloscope. Figure 23 shows rf spectra of the electrical and optical outputs of RTD-LD circuits I and II of Fig. 20(b), both dc biased close to the valley region. Figure 23(a) confirms the pulse nature of the current relaxation oscillations with a high harmonic content up to $12^{\text {th }}$ harmonic being measured.

Tuning the dc bias across the NDC region changes the RTD impedance and as consequence tunes the relaxation oscillation frequency making the circuit operate as a voltage controlled oscillator (VCO). Since the current relaxation oscillation waveforms flow through the laser diode, the circuit optical output emulates the current oscillations. The laser output shows the same repetitive switching and harmonic content of the relaxation oscillation current waveforms, making the RTD-LD circuit operate as an optoelectronic voltage controlled oscillator (OVCO). That is, the RTD-LD biased on the NDC region produces electrical and 


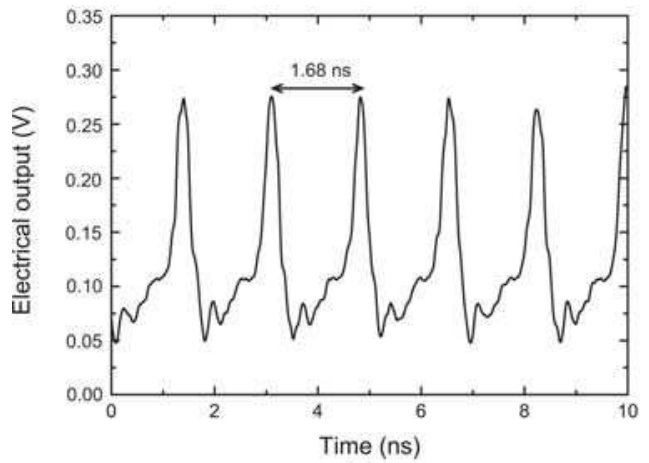

(a)

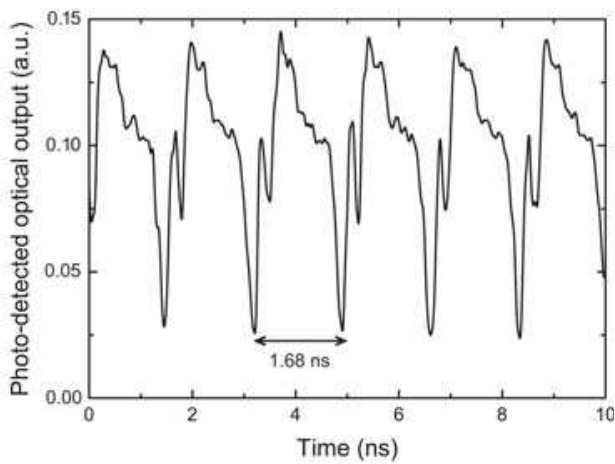

(b)

Fig. 22. RTD-LD I relaxation oscillation (a) electrical and (b) photo-detected optical output waveforms at around $600 \mathrm{MHz}$.

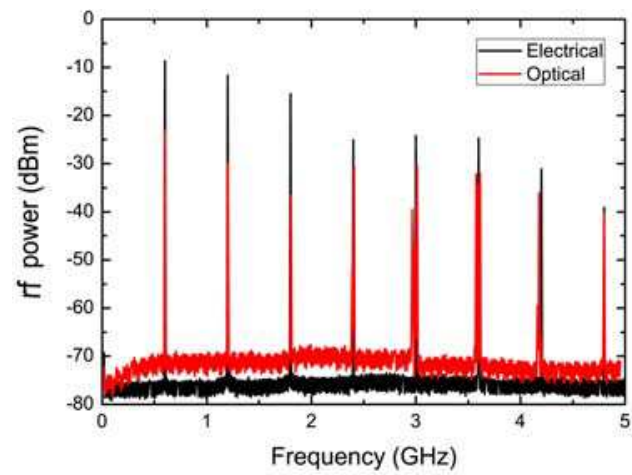

(a)

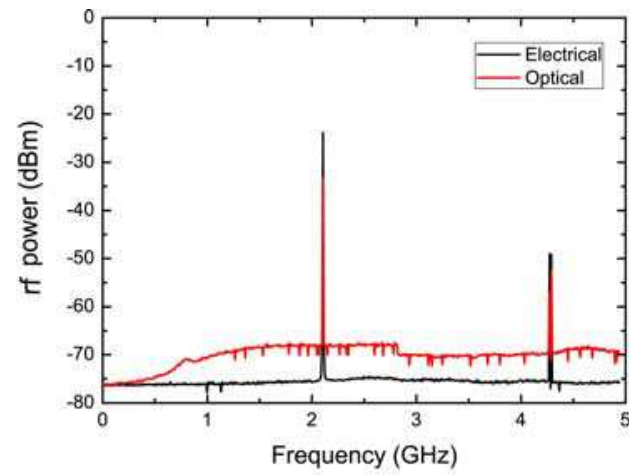

(b)

Fig. 23. Electrical and photo-detected optical spectra of free-running oscillations at $600 \mathrm{MHz}$ (a) and $2.1 \mathrm{GHz}$ (b), circuits I and II, respectively.

optical oscillatory signals whose frequency is controlled by the bias voltage quiescent point. Figure 24 shows the frequency response to dc voltage sweep across the NDC region of circuits RTD-LD I and II, whose $I-V$ characteristics are presented in Fig. 20(b).

The oscillation frequency of circuit I changed with the dc voltage from around $500 \mathrm{MHz}$ to $640 \mathrm{MHz}$, that is, RTD-LD I had a tuning range around $140 \mathrm{MHz}$, whereas the circuit II oscillate from $1.97 \mathrm{GHz}$ to $2.15 \mathrm{GHz}$, i.e., RTD-LD II had a tuning range around $180 \mathrm{MHz}$. Although the dc voltage tuning of circuit I was larger, the tuning sensitivity/tuning performance expressed in tuning range per voltage range was higher for circuit II. In the RTD-LD oscillators analyzed, we found that a linear deviation characteristic is attained considering only voltages close to the peak voltage. The voltage tuning range of circuit I, Fig 24(a), is much larger than the circuit II, Fig. 24(b), as expected from higher PVVR measured in the $I-V$ characteristic. Frequency tuning ranges up to $450 \mathrm{MHz}$ were observed in RTD-LD circuits having NDC widths and $I-V$ characteristics identical to RTD-LD I. Generally speaking, to have a wide dc operating range and therefore large tunability, a wide negative conductance region (large difference between the peak and valley voltage) is required. 


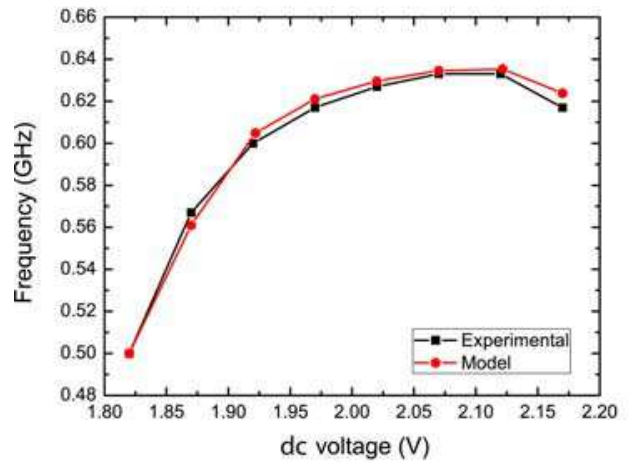

(a)

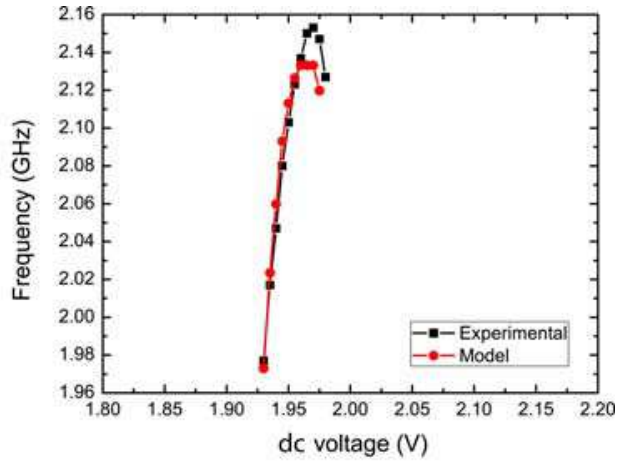

(b)

Fig. 24. RTD-LD I (a) and RTD-LD II (b) experimental and simulated frequency tuning responses to voltage sweeping across the NDC regions.

The RTD-LD optoelectronic voltage controlled oscillator is a simple way to convert fast, short electrical pulses with low timing jitter and phase noise, into fast, sharp optical pulses.

\subsection{Phase-locking}

The injection-locking of an electrical oscillator was first described by (Van Der Pol, 1927), and the first locking bandwidth equation for electrically injection-locked oscillators was developed by (Adler, 1946), with a model based on a vacuum tube transistor. The most comprehensive theoretical review of injection-locking solid-state oscillators was given by (Kurokawa, 1973). Most of the characteristic and properties identified by the above authors can be observed with RTD-LD circuits which are much simpler oscillator configuration. When externally perturbed the RTD-LD circuit behaves as a non-autonomous oscillator (Romeirab et al. 2009), being a practical demonstration of nonlinear systems theory extensively developed over the last decades (Pikovsky et al., 2001).

Throughout the work, we observed that under appropriated bias and injection conditions the RTD-LD circuit relaxation oscillations lock to low-power injected signals that take over the oscillations, controlling the laser diode output characteristics. To investigate these locking characteristics periodic external signals at microwave frequencies were injected into the circuit. The analysis included the effects of the frequency, signal power level, and injected signal modulation formats. Phase-locking with significant noise reduction to low power signals (below $-30 \mathrm{dBm}$ ) at frequencies around the circuits' natural frequencies are observed. Figure 25(a) presents rf spectra of photo-detected laser optical outputs when the circuit was free-runing at $600 \mathrm{MHz}$ and when phase-locked to $-25 \mathrm{dBm}$ power rf signal also at $600 \mathrm{MHz}$. The single side band (SSB) phase noise measurement showed the oscillation noise at $10 \mathrm{kHz}$ offset was reduced by about $35 \mathrm{~dB}$ due to the phase-locking. For the conditions of Fig. 25(a) the locking range was $1.8 \mathrm{MHz}$. The frequency locking range increases as the injected power rises, as shown in Fig. 25(b). This behavior is well described by the optoelectronic model presented previously and is represented by the red zone of Fig. 25(b), known as Arnold tongue. Arnold tongues correspond to synchronization regions were locking occurs between two competing frequencies (Pikovsky et al., 2001). When the injected signal frequency becomes out of the oscillator locking range, the circuit generate mixing products of the injected signal and free-running oscillations. 
Since the phase of a signal plays an important role in communications, particularly wireless communication, and in the theory of synchronisation, we investigated the effect of phase modulation in the RTD-LD outputs. Figure 25(c) shows circuit response to an injected 600 $\mathrm{MHz}$ carrier phase modulated with $1 \mathrm{MHz}$ frequency sub-carrier with phase shift $\pi$ and $3 \pi / 2$. As the sub-carrier frequency was varied from $100 \mathrm{kHz}$ up to $2 \mathrm{MHz}$, the laser output followed the phase modulation of the sine-wave signal subcarrier.

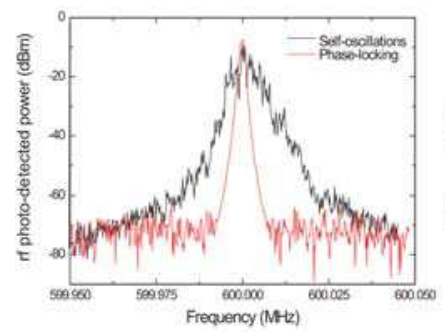

(a)

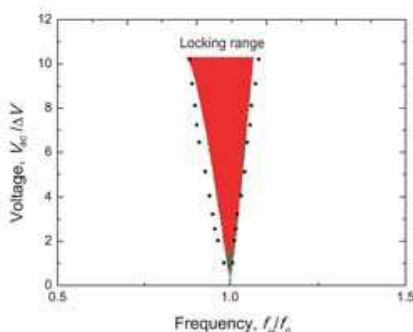

(b)

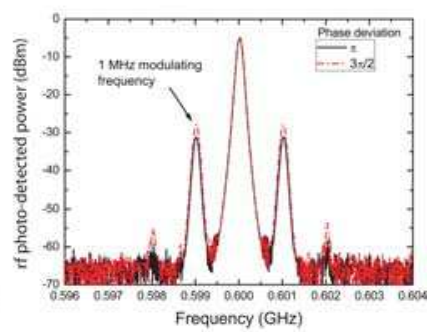

(c)

Fig. 25. (a) Rf spectra of photo-detected laser output in free-running mode and when phaselocked to $-25 \mathrm{dBm}$ injected signal at $600 \mathrm{MHz}$ frequency. (b) Frequency locking range as function of the injected power. The dotted points are experimental data and the red area (Arnold tongue) was numerically obtained. (c) Rf spectra of photo-detected laser output when phase-locking to a phase modulated $600 \mathrm{MHz}$ sine-wave carrier signal.

The observed phase-locking converts phase differences on shifts in the laser output modulating its intensity. This behaviour can be applied to implement phase shift keying (PSK) digital modulation, which is employed in numerous digital communication systems. The phase-locking capabilities of RTD-LD based relaxation oscillators can also be used for error free timing extraction in optoelectronic circuits.

\subsection{Frequency division operation}

When the injected signal frequency is out of the oscillator locking range the circuit generates mixing products of the injected signal and free-running oscillations, producing either/ both harmonic and sub-harmonic phase-locking. To investigate the mixing capability of the circuit we analysed numerically the behaviour of the circuit over a range of frequencies to obtain the laser optical output bifurcation diagram of Fig. 26. A bifurcation diagram shows the amplitude peaks heights of output photon density oscillations, $S$, as a function of the normalized excitation frequency $f_{\text {in }} / f_{0}$, where $f_{0}$ is the free running oscillation frequency. The simulation results show that when the frequency of the injected signal, $f_{i n}$ is successively increased, a stable period $-n, n=1,2, \ldots$ is obtained, followed by an unlocked region, then a stable period- $(n+1)$, a new unlocked region and so on (Figueiredo et al., 2008)(Pikovsky et al., 2001). This phenomenon is known as period-adding, where windows of consecutive regions showing frequency division are separated by zones of unlocked, even chaotic, signals. The frequency division regions were obtained experimentally and calculated numerically dc biasing the RTD-LD circuit on the NDC region and varying the frequency of the injected signal from $0.1 \mathrm{GHz}$ to $3 \mathrm{GHz}$, with drive amplitudes as low as $100 \mathrm{mV}$. Frequency division regions for constant amplitudes were observed following the period- 
adding sequence at up to frequency division by 6 . In Fig. 26(a) the period-adding is clearly distinguished in a sequence of unlocked (dots) and periodic (branch regions) oscillations, as observed experimentally. Figure 26(b) presents an experimental example of frequency division by 2 when a $0.9 \mathrm{GHz}$ sine-wave was injected.

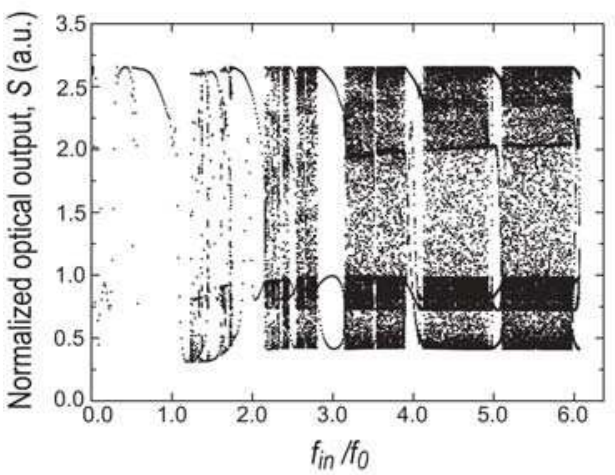

(a)

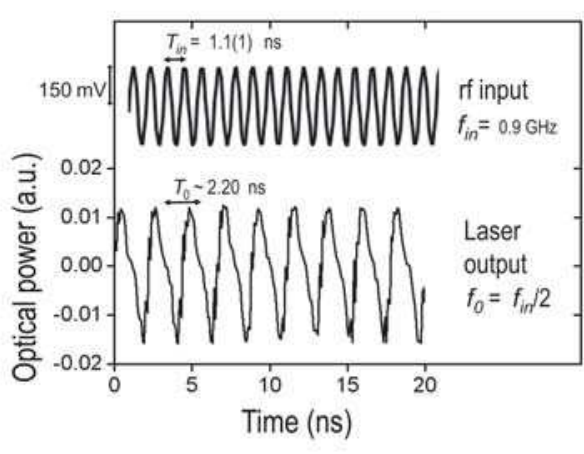

(b)

Fig. 26. (a) Calculated bifurcation diagram for $V_{a c}=150 \mathrm{mV}$ up to frequency division by 6 .

(b) Photo-detected laser output showing frequency division by 2 when a signal with $f_{\text {in }}=0.9$ $\mathrm{GHz}$ was injected into an RTD-LD free-running oscillating at around $0.5 \mathrm{GHz}$.

Since the sub-harmonic windows appear in limited frequency regions, the RTD-LD circuit can be regarded as an optoelectronic dynamic frequency divider with a selectable dividing ratio.

\subsection{Aperiodic and chaotic operation}

Electro-optical and all-optical solutions for complex chaos generation have attracted considerable attention in the last decade due to their potential applications in optical chaos communications (Argyris et al., 2005). The use of chaotic carriers allows steganography at the physical layer, which can substantially improve the security of software encryption techniques. The frequency bands corresponding to period multiplication, indicated in Fig. 26(a), are separated by frequency regions where the circuit generates aperiodic signals chaotic or quasi-periodic output - a direct result from the mixing between free-running oscillation and external injected frequencies (Romeira et al. 2010). An important characteristic of a chaotic signal is its sensitivity to initial conditions. Figure 27 shows an example of a transition to chaos observed in the RTD-LD circuit optical output. The optical waveform presented in Fig. 27(a) is characterized by a series of aperiodic acute peaks (spikes) changing chaotically. Another important characteristic of chaos is demonstrated in the corresponding power spectrum of the time series. Figure 27(b) shows a continuous and broadband spectrum resembling a noisy process with a few dominant frequencies appearing, in this case the rf injected frequency. The results of Fig. 27 are also confirmed numerically by calculating the circuit Lyapunov exponents (Romeira et al., 2001).

This RTD-LD mode of operation provides a simply way to generate and convert electrical chaotic signals into optical sub-carriers that can be transmitted by conventional optical 
channels. Moreover, the circuit allows direct addition of the message to be transmitted and masked within the chaotic signal.

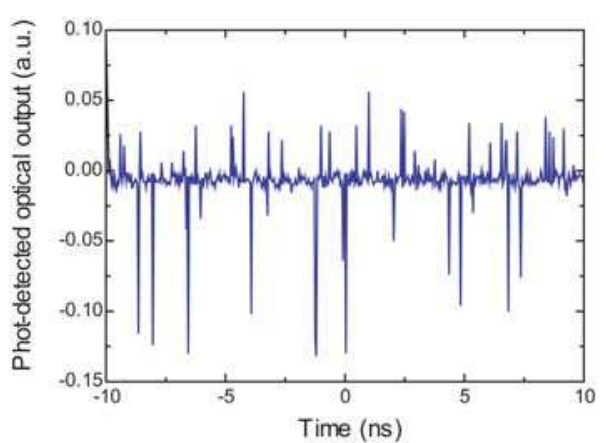

(a)

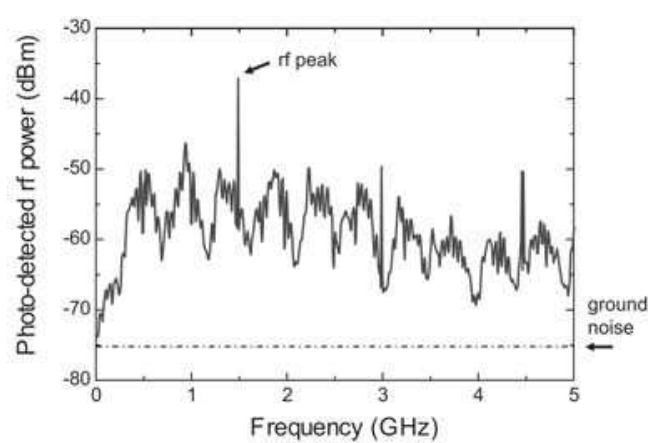

(b)

Fig. 27. Chaotic behaviour in the laser output induced by a driving signal of frequency $1.485 \mathrm{GHz}$ and amplitude $793 \mathrm{mV}$. Optical waverform (a) and corresponding Fourier spectrum (b).

\section{Conclusion}

As discussed, embedding DBQW-RTDs within semiconductor optical waveguides can lead to the implementation of highly efficient electro-absorption modulators and photo-detectors operating at optical wavelengths around $900 \mathrm{~nm}$ and $1550 \mathrm{~nm}$. The presence of the DBQW introduces high non-linearities and NDC regions in the semiconductor optical waveguides current-voltage characteristics, making the electric field distribution across the waveguide core strongly dependent on the bias voltage, which can be used to modulate guided light through the Franz-Keldysh electro-absorption effect. When biased on the NDC region the RTD-OW operates as an optoelectronic voltage controlled oscillator. Electro-absorption modulation up to $28 \mathrm{~dB}$ is achieved with high frequency signals as low as $100 \mathrm{mV}$. The key difference between these RTD-OW electro-absorption modulators and conventional $p-i-n$ electro-absorption modulators is that the RTD-EAM has in essence an integrated electronic amplifier and therefore requires considerably less switching/driving power. Since, the RTDOWs can also work as photo-detectors with built-in amplifiers, recovering the original transmitted rf signals used to modulated the optical carriers, they can be employed at the base station to convert information from the optical to the rf domains. We foresee that optimized devices can have bandwidths up to $60 \mathrm{GHz}$.

By integrating a DBQW-RTD with a laser diode low-cost microwave-photonic circuits operating up to $2.15 \mathrm{GHz}$ were implemented. These circuits reduced significantly the driving circuitry of laser diodes. Several optoelectronic operation modes were observed, including optoelectronic voltage controlled oscillator (OVCO), phase-locking, frequency division and generation of aperiodic electrical and optical waveforms. Their simple circuit layout is appropriated for high functional single chip transmitter platforms due to their nonlinear optoelectronic characteristics, reduced size and low power consumption. We 
anticipate that the optimised RTD-LD monolithic integrated versions can operate at much higher frequencies (tens of Gbits), having several advantages when compared to conventional devices currently used in lightwave communication systems.

The RTD-OW and RTD-LD operation as optoelectronic voltage controlled oscillators can be used to simplify significantly clock generation and clock extraction circuits. Due to the nonlinear response to applied voltage the RTD based circuits can work as short optical pulse generators with high repetition rates. At the same time, their integration with other functional devices can be used to encode generated optical pulses. The combination of RTDOW and RTD-LD functions on a single circuit can be used to incorporate simultaneously rf subcarrier signals into optical carriers and optical subcarrier signals into rf carriers. This is possible due the following simultaneously capabilities: modulation, photo-detection and intrinsic amplification. Thus, the RTD-OW and RTD-LD circuits offer the possibility of implementing very simple microwave/photonics interfaces of cellular network terminal base stations based on radio-over-fiber systems.

Since next generation wireless access picocellular networks will be based on large numbers of short range cells with each office in a building with its own cells and base stations, the RTD based optoelectronic devices offer low cost single chip solutions as microwave/optical interfaces capable of electrical-to-optical conversion of microwave signals into optical subcarriers, taking advantage of the NDC and phase-locking properties of RTD devices. The photo-detecting capabilities allows recovery of the original transmitted rf signals used to modulated the optical carriers sent from the office terminal station to each base station via optical fibre, converting the information from the optical to the rf domain; light generation function is used to transfer the wireless received information bearing signals from the rf domain to optical domain which is then sent from the base stations to the office terminal station via optical fibre.

\section{Acknowledgment}

Bruno Romeira and José Figueiredo acknowledge the support of the Centro de Electrónica, Optoelectrónica e Telecomunicações, Portugal. This work was also supported in part by the Fundação para a Ciência e a Tecnologia, Portugal, through the grants PRAXIS XXI/BD/2871/94 and SFRH/BD/43433/2008, by the Fundação Calouste Gulbenkian, Portugal, and by Research Networks - Treaty of Windsor Programme 2008/09-U32, Portugal. The authors would like to thank W. Meredith of Compound Semiconductor Technologies Global, Ltd. for providing the laser diodes, and Liquan Wang and Edward Wasige by the fruitful discussions and PCB layout design in the RTDLD work.

\section{References}

Adler, R. (1946). A study of locking phenomena in oscillators. Proc. I.R.E.Waves Electrons, 351- 357

Alkeev, N. V.; Lyubchenko, V. E.; Ironside, C. N.; Figueiredo, J. M. L. \& Stanley C. R. (2000) Super high-frequency characteristics of optical modulators on the basis of 
InGaAlAs resonance-tunnel heterostructures. J. Commun. Technol. Electron., Vol. 45, No. 8, Aug. 2000, 911-914

Argyris, A.; Syvridis, D.; Larger, L.; Annovazzi-Lodi, V.; Colet, P.; Fisher, I.; Garcia-Ojalvo, J.; Mirasso, C.; Pesquera, L.; Shore, K. A. (2005). Chaos-based communications at high bit rates using commercial fibre-optic links. Nature, Vol. 43, Nov. 2005, 343346

Bohm, D. (1951). Quantum theory, Prentice-Hall Inc., New Jersey

Böhrer, J.; Krost, A. \& Bimberg, D. B. (1993). Composition dependence of band gap and type of lineup in InGaAlAs/InP heterostructures. Appl. Phys. Lett., Vol. 63, No. 14, Oct. 1993,1918

Brown, E. R.; McMahon, O. B.; Mahoney, L. J. \& Molvar, K. M. (1996). SPICE model of the resonant-tunneling diode. Electronics Lett., Vol. 32, No. 10, May 1996, 938-940

Brown, E. R. \& Parker C. D. (1996). Resonant tunnel diodes as submillimetre-wave sources. Phil. Trans. R. Soc. Lon. A, Vol. 354 , No. 1717, Oct. 1996, 2365-2381

Brown, E. R.; Parker, C. D.; Verghese, S. \& Geis, M. W. (1997). Resonant-tunneling transmission-line relaxation oscillator. Appl. Phys. Lett., Vol. 70, No. 21, May 1997, 2787

Capasso, F.; Mohammed, K. \& Cho A. Y. (1986). Resonant tunneling through double barriers, perpendicular quantum transport phenomena in superlattices, and their device applications. IEEE J. Quantum Electron., Vol. 22, No. 9, Sep. 1986, $1853-$ 1869

Chang, L. L.; Esaki, L. \& Tsu, R. (1974). Resonant tunneling in semiconductor double barriers. Appl. Phys. Lett., Vol, 24, No. 12, Jun. 1974, 593

Chen, L.; Kapre, R. M.; Hu, K. \& Madhukar, A. (1991). High-contrast optically bistable optoelectronic switch based on InGaAs/GaAs (100) asymmetric Fabry-Perot modulator, detector, and resonant tunneling diode. Appl. Phys. Lett., Vol. 59, No. 13, Sep. 1991, 1523-1525

Chuang, S. L. (1995). Physics of optoelectronic devices JohnWiley \& Sons Inc., New York

Davies, J. H. (1998). The physics of low-dimensional semiconductors: an introduction, Cambridge University Press, Cambridge.

Einarsson, G. (1996). Principles of lightwave communications, John Wiley \& Sons, New York

England, P.; Golub, J. E.; Florez, L. T. \& Harbison, J. P. (1991). Optical switching in a resonant tunneling structure. Appl. Phys. Lett., Vol.58, No. 9, Mar. 1991, 887-889

Figueiredo, R. (1970). Existence and Uniqueness results for Liénards equation. IEEE Transactions on Circuit Theory, Vol. CT-17, No. 3, Jun. 1970, 313-321

Figueiredo, J. M. L.; Boyd, A. R.; Stanley, C. R.; Ironside, C. N.; McMeekin, S. G. \& Leite, A. M. P. (1999). Optical modulation at around $1550 \mathrm{~nm}$ in an InGaAlAs optical waveguide containing an InGaAs/AlAs resonant tunneling diode. Appl. Phys.Letts, Vol. 75, No. 22, Nov. 1999, 3443-3445 
Figueiredo, J. M. L. (2000). Optoelectronic Properties of Resonant Tunneling Diodes, PhD Thesis, Universidade do Porto, Porto

Figueiredo, J.M.L.; Stanley C.R. \& Ironside C.N. (2001). Electric Field Switching in a Resonant Tunneling Diode Electroabsorption Modulator. IEEE Journal of Quantum Electronics, Vol. 37, No. 12, Dec. 2001, 1547-1552

Figueiredo, J. M. L.; Ironside C. N. \& Stanley. C. R.; (2002). Ultralow voltage resonant tunneling diode electroabsorption modulator. Journal of Modern Optics, Vol. 49, No. 5, Apr. 2002, 939-945

Figueiredo, J. M. L.; Romeira, B.; Slight, T. J.;Wang, L.;Wasige E. \& Ironside C. N. (2008). Selfoscillation and period adding from a resonant tunnelling diode laser diode circuit. Electronics Letters, Vol. 44, No. 14, Jul. 2008, 876-878

Gravé, I.; Kan, S. C.; Griffel, G.; Wu, S. W.; Sa'ar, A. \& Yariv, A. (1991). Monolithic integration of a resonant tunneling diode and a quantum well semiconductor laser. Appl. Phys. Lett. Vol. 58, No. 2, Jan. 1991, 110-112

Huang, C. Y.; Morris, J. E. \& Su, Y. K. (1997). Generalized formula for the stability and instability criteria of current-voltage characteristics measurements in the negative differential conductance region of a resonant tunneling diode. J. Appl. Phys., Vol. 82, No. 5, Sep 1997, 2690

Iogansen, L. V. (1964). The possibility of resonance transmission of electrons in crystals through a system of barriers. Soviet Physics JETP, Vol. 18, 146

Katz, A. (1992). Indium phosphide and related materials: processing, technology, and devices, Artech House, London

Kawamura, Y.; Wakita, K.; Asai, H. \& Oe, K. (1987). Optically bistable operation in InGaAsInAlAs MQW laser diodes using resonant tunnelling effect. Electron. Lett., Vol. 23, No. $14,719-721$

Kawamura, Y.; Wakita, K. \& Mikami, O. (1988). InGaAs/InAlAs bistable multiple quantum well lasers with large on/off ratio using the resonant tunneling effect. Appl. Phys. Lett., Vol.53, No. 16, Oct. 1986, 1462-1464

Kawamura, Y.; Asai, H.; Matsuo, S. \& Amaro, C. (1992). InGaAs-InAlAs multiple quantum well optical bistable devices using the resonant tunneling effect. IEEE J. Quantum Electron., Vol. 28, No. 1, Jan. 1992, 308-314

Kawamura, Y.; Asai, H. \& Iwamura, H. (1994). Fabrication of resonant-tunnelling optical bistable laser diodes. Electron. Lett., Vol. 30, No. 3, Feb. 1994. 225-227

Keldysh, L. V. (1958). The effect of a strong electric field on the optical properties of insulating crystals. Sov. Phys. JETP, Vol. 34, 788

Kidner, C.; Mehdi, I.; East, J. R. \& Haddad, G. I. (1990). Power and stability limitations of resonant tunneling diodes. IEEE Trans. Microwave Theory and Techniq., Vol. 38, No. 7, Jul 1990, 864-872

Kurokawa, K. (1973). Injection locking of microwave solid-state oscillators. Proc. IEEE, Vol. 61, No. 10, Oct. 1973, 1386-1410

Lann, A. F.; Grumann, E.; Gabai, A.; Golub, J. E.; \& England, P. (1993). Phase locking between light pulses and a resonant tunneling diode oscillator. Appl. Phys. Lett., Vol. 62, No. 1, Jn. 1993, 13-15 
Liu, M. M.-K. (1996). Principles and applications of optical communications, Irwin Book, London.

Mazumder, P.; Kulkarni, S.; Bhattacharya, M.; Sun, J. P. \& Haddad, G. I. (1998). Digital circuit applications of resonant tunneling devices. Proceedings of the IEEE, Vol. 86, No. 4, Apr. 1998, 664-686

McMeekin, S. G.; Taylor, M. R. S.; V“ogele, B.; Stanley, C. R. \& Ironside, C. N. (1994). FranzKeldysh effect in an optical waveguide containing a resonant tunneling diode, Appl. Phys. Lett., Vol 65, No. 9, Aug. 1994, 1076-1078

Mizuta, H. \& Tanoue, T. (1995). The physics and applications of resonant tunneling diodes, Cambridge University Press, Cambridge

Moise, T. S.; Kao, Y.-C.; Garrett, L. D. \& Campbell, J. C. (1995). Optically switched resonant tunneling diodes. Appl. Phys. Lett., Vol. 66, No. 9, Feb. 1995, 1104-1106

Moise, T. S.; Kao, Y.-C.; Goldsmith, C. L.; Schow, C. L. \& Campbell, J. C. (1997). High-speed resonant-tunneling photodetectors with low-switching energy. IEEE Photonics Technol. Lett., Vol. 9, No. 6, Jun. 1997, 803-805

Park, T. J.; Lee, Y. K.; Kwon, S. K.; Kwon,J. H. \& Jang, J. (2006). Resonant tunneling diode made of organic semiconductor superlattice. Appl. Phys. Lett. Vol. 89, No. 15, Oct. 2006, 151114

Pikovsky, A.; Rosenblum, M. \& Kurths J. (2001). Synchronization: A universal concept in nonlinear sciences, Cambridge Univ. Press,Cambridge

Romeira, B.; Figueiredo, J. M. L.; Slight, T. J.; Wang, L.; Wasige, E.; Ironside C. N.; Quintana, J. M. \& Avedillo, M. J. (2008). Synchronisation and chaos in a laser diode driven by a resonant tunneling diode. IET Optoelectronics, Vol. 2, No. 6, Dec. 2008, 211-215

Romeiraa, B.; Figueiredo, J. M. L.; Slight, T. J.; Wang, L.; Wasige, E. \& Ironside C. N. (2009) Wireless/Photonics Interfaces Based on Resonant Tunneling Diode Optoelectronic Oscillators, Conference on Lasers and Electro-Optics (CLEO)/The International Quantum Electronics Conference (IQEC), paper CTuT4, Baltimore, Maryland, USA, May 31June 5, 2009, OSA Technical Digest (CD) Optical Society of America

Romeirab, B.; Figueiredo, J. M. L.; Slight, T. J.; Wang, L.; Wasige, E.; Ironside, C. N.; Kelly, A. E. \& Green, R. (2009). Nonlinear Dynamics of Resonant Tunneling Optoelectronic Circuits for Wireless/Optical Interfaces. IEEE J. Quantum Electron., Vol. 45, No. 11, Nov. 2009, 1436-1445)

Romeira, B.; Figueiredo, J. M. L.; Ironside, C. N. \& Slight, T. J. (2010). Chaotic Dynamics in Resonant Tunneling Optoelectronic Voltage Controlled Oscillators. to be published in IEEE Photonics Technol. Lett. DOI: 10.1109/LPT.2009.2034129

Ryu, S. Y.; Jo, S. J.; Kim, C. S.; Choi, S. H.; Noh, J. H.; Baik, H. K.; Jeong, H. S.; Han, D.W. Song, S. Y. \& Lee, K. S. (2007). Transparent organic light-emitting diodes using resonant tunneling double barrier structures. Appl. Phys. Lett. Vol. 91, No. 9, Aug. 2007, 093515

Sakata, H.; Utaka, K. \& Matsushima, Y. (1995). Diferent types of optoelectronic bistabilities in resonant-tunnelling triangular-barrier optoelectronic switch (R-TOPS). Electron. Lett., Vol. 31, No. 13,Jun 1995, 1098-1099 
Sauer, M.; Kobyakov, A. \& George, J. (2007). Radio over fiber for picocellular network architectures. J. Lightw. Technol., Vol. 25, No. 11, Nov. 2007, 3301-3320

Sano, K.; Murata, K.; Akeyoshi, T.; Shimizu, N.; Otsuji, T.; Yamamoto, M.; Ishibashi T. \& Sano, E. (1998). Ultra-fast optoelectronic circuit using resonant tunnelling diodes and uni-travelling-carrier photodiode. Electron. Lett., Vol 34, No. 2, Jan. 1998, $215-$ 217

Schulman, J. N.; De Los Santos, H. J. \& Chow, D. H. (1996). Physics-based RTD current-voltage equation. IEEE Electron Device Lett., Vol. 17, No. 5. May 1996, 220 223

Slight, T. J.; Ironside, C. N.; Stanley, C. R.; Hopkinson, M. \& Farmer, C. D. (2006). Integration of a resonant tunneling diode and an optical communications laser. IEEE Photonics Technology Letters, Vol. 18, No. 14, Jul. 2006, 1518-1520

Slight, T. J. (2006). Integration of a Resonant Tunnelling Diode and an Optical Communications Laser, PhD Thesis, University of Glasgow, Glasgow

Slight, T. J. \& Ironside, C. N. (2007). Investigation into the integration of a resonant tunneling diode and an optical communications laser: model and experiment. IEEE J. Quant. Elec. Vol. 43, No. 7, Jul 2007, 580-587

Slight, T. J.; Romeira, B.; Wang, L.; Figueiredo, J. M. L.; Wasige E., \& Ironside, C. N. (2008). A Liénard oscillator resonant tunnelling diode-laser diode hybrid integrated circuit: model an experiment. IEEE J. Quantum Electron., vol. 44, no. 12, Dec. 2008, 11581163

Sollner, T. C. L. G.; Goodhue, W. D.; Tannenwald, P. E.; Parker, C. D. \& Peck, D. D. (1983). Resonant tunneling through quantum wells at frequencies up to $2.5 \mathrm{THz}$. Appl. Phys. Lett., Vol. 43, No. 3, Sep. 1983, 588-590

Sun, J. P.; Haddad, G. I.; Mazumder, P. \& Schulman, J. N. (1998). Resonant tunneling diodes: models and properties. Proceedings of the IEEE, Vol. 86, No. 4, Apr. 1998, 641-660

Suzuki, S.; Teranishi, A.; Hinata, K.; Asada, M.; Sugiyama H. \& Yokoyama H. (2009). Fundamental Oscillation of up to $831 \mathrm{GHz}$ in GaInAs/AlAs Resonant Tunneling Diode. Appl. Phys. Express, Vol. 2, Apr. 2009, 054501

Tsu, R. \& Esaki, L. (1973). Tunneling in a finite superlattice. Appl. Phys. Lett., Vol. 22, No. 11, Jun. 1973, 562-564

Wada, O. (1994). Optoelectronic integration: physics, technology and applications, Kluwer Academic Publishers, Massachusetts

Wakita, K. (1998). Semiconductor optical modulators, Kluwer Academic Publishers, London

Weisbuch, C. \& Vinter, B. (1991). Quantum semiconductor structures: fundamentals and applications, Academic Press Inc., London

Van Der Pol, (1927). Forced Oscillator in a circuit with nonlinear resistance. Phil. Mag. Vol. 3, 65-80

Van Hoof, C.; Genoe, J.; Mertens, R.; Borghs, G. \& Goovaerts, E. (1992). Electroluminescence from bipolar resonant tunneling diodes. Appl. Phys. Lett., Vol. 60, No. 1, Jan 1992, 77-79 
Van Hoof, C.; Genoe, J.; Raymond, S. \& Borghs, G. (1993). Giant optical bistable behavior using triple-barrier resonant tunnelling light-emitting diodes. Appl. Phys. Lett., Vol. 63, No. 17, Oct. 1993, 2390-2392

Zheng, T.; Choy,W. C. H. \& Sun, Y. (2009). Hybrid Nanoparticle/Organic Devices with Strong Resonant Tunneling Behaviors. Advanced Functional Materials, Vol. 19, No. 16, Jul. 2009, 2648-2653 


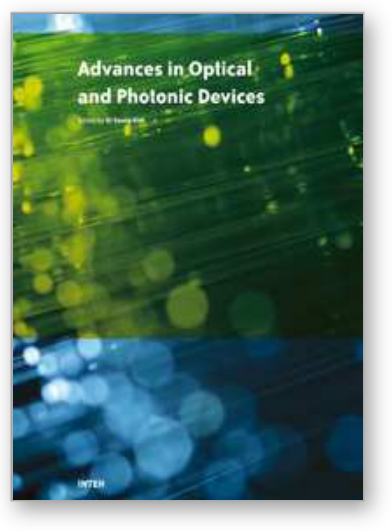

\author{
Advances in Optical and Photonic Devices \\ Edited by Ki Young Kim
}

ISBN 978-953-7619-76-3

Hard cover, 352 pages

Publisher InTech

Published online 01, January, 2010

Published in print edition January, 2010

The title of this book, Advances in Optical and Photonic Devices, encompasses a broad range of theory and applications which are of interest for diverse classes of optical and photonic devices. Unquestionably, recent successful achievements in modern optical communications and multifunctional systems have been accomplished based on composing "building blocks" of a variety of optical and photonic devices. Thus, the grasp of current trends and needs in device technology would be useful for further development of such a range of relative applications. The book is going to be a collection of contemporary researches and developments of various devices and structures in the area of optics and photonics. It is composed of 17 excellent chapters covering fundamental theory, physical operation mechanisms, fabrication and measurement techniques, and application examples. Besides, it contains comprehensive reviews of recent trends and advancements in the field. First six chapters are especially focused on diverse aspects of recent developments of lasers and related technologies, while the later chapters deal with various optical and photonic devices including waveguides, filters, oscillators, isolators, photodiodes, photomultipliers, microcavities, and so on. Although the book is a collected edition of specific technological issues, I strongly believe that the readers can obtain generous and overall ideas and knowledge of the state-of-the-art technologies in optical and photonic devices. Lastly, special words of thanks should go to all the scientists and engineers who have devoted a great deal of time to writing excellent chapters in this book.

\title{
How to reference
}

In order to correctly reference this scholarly work, feel free to copy and paste the following:

José Figueiredo, Bruno Romeira, Thomas Slight and Charles Ironside (2010). Resonant Tunnelling Optoelectronic Circuits, Advances in Optical and Photonic Devices, Ki Young Kim (Ed.), ISBN: 978-953-761976-3, InTech, Available from: http://www.intechopen.com/books/advances-in-optical-and-photonicdevices/resonant-tunnelling-optoelectronic-circuits

\section{INTECH}

open science | open minds

\section{InTech Europe}

University Campus STeP Ri

Slavka Krautzeka 83/A

51000 Rijeka, Croatia

Phone: +385 (51) 770447

\section{InTech China}

Unit 405, Office Block, Hotel Equatorial Shanghai

No.65, Yan An Road (West), Shanghai, 200040, China

中国上海市延安西路65号上海国际贵都大饭店办公楼 405 单元

Phone: +86-21-62489820 
Fax: +385 (51) 686166

Fax: +86-21-62489821

www.intechopen.com 
(C) 2010 The Author(s). Licensee IntechOpen. This chapter is distributed under the terms of the Creative Commons Attribution-NonCommercialShareAlike-3.0 License, which permits use, distribution and reproduction for non-commercial purposes, provided the original is properly cited and derivative works building on this content are distributed under the same license. 